This item was submitted to Loughborough's Research Repository by the author.

Items in Figshare are protected by copyright, with all rights reserved, unless otherwise indicated.

\title{
In-shoe pressure thresholds for people with diabetes and neuropathy at risk of ulceration: A systematic review
}

PLEASE CITE THE PUBLISHED VERSION

https://doi.org/10.1016/j.jdiacomp.2020.107815

\section{PUBLISHER}

Elsevier BV

VERSION

AM (Accepted Manuscript)

\section{PUBLISHER STATEMENT}

This paper was accepted for publication in the journal Journal of Diabetes and its Complications and the definitive published version is available at https://doi.org/10.1016/j.jdiacomp.2020.107815

\section{LICENCE}

CC BY-NC-ND 4.0

\section{REPOSITORY RECORD}

Jones, Petra, Melanie J Davies, Kamlesh Khunti, Daniel Fong, and David Webb. 2020. "In-shoe Pressure Thresholds for People with Diabetes and Neuropathy at Risk of Ulceration: A Systematic Review". Loughborough University. https://hdl.handle.net/2134/13340852.v1. 


\section{REVIEW ARTICLE}

\section{In-shoe Pressure Thresholds for People with Diabetes and} Neuropathy at Risk of Ulceration: A Systematic Review

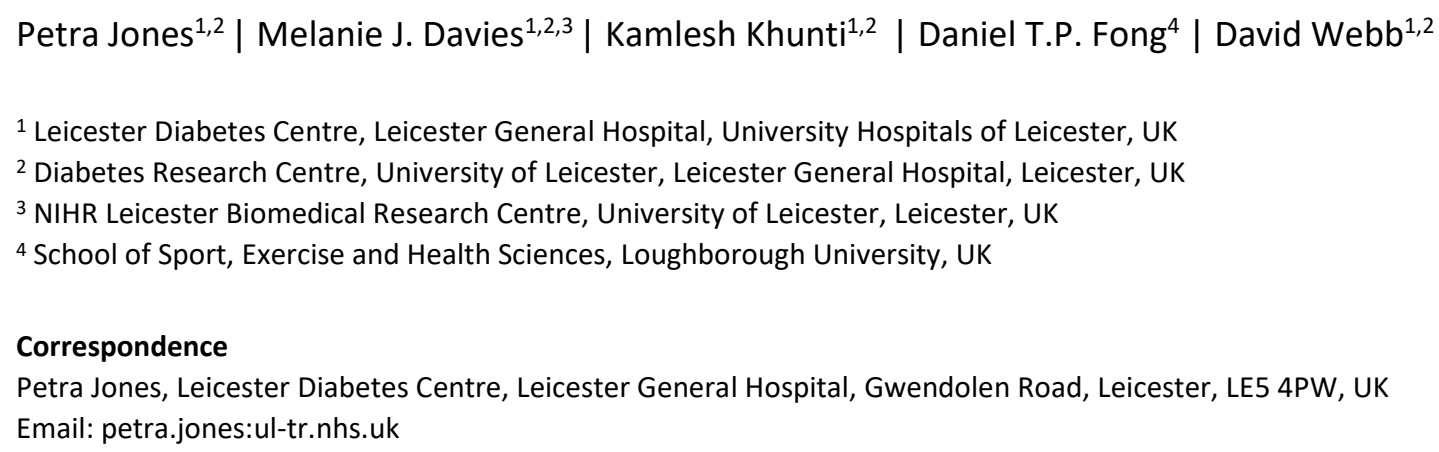

\section{Introduction}

In-shoe pressure thresholds play an increasingly important role in the prevention of diabetes-related foot ulceration (DFU). The evidence of their effectiveness, methodological consistency and scope for refinement are the subject of this review.

\section{Methods}

1,107 records were identified (after duplicate removal) based on a search of five databases for studies which applied a specific in-shoe pressure threshold to reduce the risk of ulceration. 37 full text studies were assessed for eligibility of which 21 were included.

\section{Results}

Five in-shoe pressure thresholds were identified, which are employed to reduce the risk of diabetesrelated foot ulceration: a mean peak pressure threshold of $200 \mathrm{kPa}$ used in conjunction with a $25 \%$ baseline reduction target; a sustained pressure threshold of $35 \mathrm{mmHG}$, a threshold matrix based on risk, shoe size and foot region, and a $40-80 \%$ baseline pressure reduction target. The effectiveness of the latter two thresholds have not been assessed yet and the evidence for the effectiveness of the other in-shoe pressure thresholds is limited, based only on two RCTs and two cohort studies.

\section{Conclusions}

The heterogeneity of current measures precludes meta-analysis and further research and methodological standardisation is required to facilitate ready comparison and the further development of these pressure thresholds.

\section{KEYWORDS}

diabetes, foot, neuropathy, in-shoe, pressure, threshold 


\section{Abbreviations}

53

54

BMI, Body mass index; CG, Control group, without diabetes; D, Diabetes without neuropathy; DFU, Diabetes-related foot ulcer; DN, Diabetes and neuropathy; DN+HU, Diabetes, neuropathy and a history of foot ulceration; $\mathrm{kPa}$, Kilopascals, a measurement unit of pressure; $\mathrm{mmHG}$, Millimetres of Mercury, an alternative measurement unit of pressure; LOPS, Loss of protective sensation associated with neuropathy; MTH, Metatarsal heads; MTP, Metatarsophalangeal joint; N/ $\mathrm{cm}^{2}$, Newtons, an alternative measurement unit of pressure for a defined area in centimetres; SD, Standard deviation; VPT, Vibration perception threshold.

\section{Introduction}

Globally 463 million adults are currently living with diabetes which is predicted to rise to 700 million by 2045 [1]. Among these, 15\%-25\% [2, 3] are likely to develop a foot ulcer within their lifetime. The financial burden of diabetes-related foot ulceration (DFU) is immense: an estimated $\$ 9-13$ billion [4] and up to $f 1.13$ billion per year [5] is spent on treatment in the United States and UK alone. DFU is also associated with an increased risk of cardiovascular mortality [6] and amputation [7]. An estimated $40 \%$ of ulcers will reoccur within a year after healing, which increases to almost $60 \%$ within three years [8].

Predicting ulceration events remains problematic given their multi-factorial nature [9] where the degree of peripheral vascular disease and neuropathy, foot shape and deformity, altered Achilles reflex and footwear may all be implicated. Development of a threshold or set of thresholds to either predict or at least minimise the risks of DFU is important given five-year mortality rates after newonset diabetic ulceration ranging between $43 \%$ and $55 \%$ [10]. As many as one-in-three individuals with neuropathic DFU will have an amputation [11].

A particularly active area of research concerns ulcer prevention through assessment of in-shoe plantar pressures, given that people with diabetes and neuropathy have increased mean plantar pressures [12-17] in comparison with those with healthy feet (see Supplemental Table 1). These pressures are elevated further in those with a history of ulceration as well as diabetes and neuropathy [16, 18-19]. Elevated plantar pressures may be linked to a variety of factors including deformity [20], limited joint mobility [21] and ankle equinus [22], soft tissue glycation [23], shortening of the Achilles tendon [24] or disorganisation of its tendon fibres [25-26] and a reduction in cross-sectional muscle area [27].

A review of pressure thresholds is also important because peripheral neuropathy limits the ability to detect abnormal high pressures within footwear. In-shoe pressure monitoring technology offers potential both to determine thresholds associated with an increased risk of ulceration and a means of alerting individuals with neuropathy to their presence. In-shoe pressure analysis is also playing a growing role in footwear, insole and orthotic design and may also come have a future role to play in fit, having been used to evaluate the role toe box shape [28]. There are no systematic reviews on 
this topic as yet as previous reviews are narrative [29] and the range of available wearable devices is growing with a corresponding expanding literature that requires synthesis.

In this systematic review, we examine the literature around specific in-shoe pressure thresholds to prevent diabetes-related foot ulceration.

\section{Methods}

\subsection{Search strategy}

The Preferred Reporting Items for Systematic Reviews and Meta-Analyses (PRISMA) guidelines were used to perform this systematic review. [30] This involved defining the population of interest, which includes people with diabetes mellitus (type 1 or type 2 ) with neuropathy and either a history of foot ulceration or those at risk of ulceration. By risk of ulceration, we refer to the International Working Group on the Diabetic Foot (IWGDF) definition of an at-risk person as a 'person with diabetes who does not have an active foot ulcer, but who has peripheral neuropathy, with or without the presence of foot deformity or peripheral artery disease, or a history of foot ulcer(s) or amputation of (a part of) the foot or leg' [31].

We searched Medline/Pubmed, Scopus, Google Scholar and Web of Science databases for all studies ever published until 1 September 2020 using the search criteria "pressure" AND "foot" AND "inshoe" AND "diabetes" AND "threshold" across all databases. In addition, a further search was carried out on all these databases with no start date specified through to 3 September 2020 which used the search terms "plantar" AND "pressure" AND "foot" AND "in-shoe" AND "diabetes" AND "threshold".

\subsection{Inclusion Criteria}

Studies were included if they fulfilled the following selection criteria: (i) Studies which a priori involved the application of specific in-shoe plantar pressure thresholds in an intervention intended to prevent DFU; (ii) Type of study: RCT, cohort, or case-control studies published within peerreviewed journals; (iii) Results: studies which evaluated pressure reduction through an intervention related to the use of in-shoe pressure thresholds and DFU; and (iv) published in the English language. We concentrated on in-shoe rather than barefoot pressure thresholds given people with diabetes and neuropathy spend most of their time wearing footwear and engaging in physical activity during which foot inflammation, calluses or trauma may arise. Poor footwear fit is considered a risk factor for foot injury and ulceration, notably at the toes, or metatarsals [32]. The review does not include thresholds for healed Charcot foot, amputation or post-surgery.

\subsection{Data extraction}

Data extracted was performed by PJ. This included (i) Article identification: title, authors and publication year; (ii) Methods: sample size and demographic (e.g. history of ulceration, neuropathy), loss of protective sensation testing to establish neuropathy, in-shoe pressure monitoring methodology including pace, number of steps, sensor type and standardisation of footwear and socks, plantar region of interest and pressure threshold criteria; (iii) Outcome: objective and type of intervention; (iv) Results: methods of statistical analysis and degree of statistical significance (Tables 1-3). DW checked the extracted data and was consulted regarding queries on whether to include or exclude particular studies with MJD available to resolve any disagreements. 
P. Jones

v2-0b

141

142 

$(n=2,370)$ references within identified papers $(n=5)$

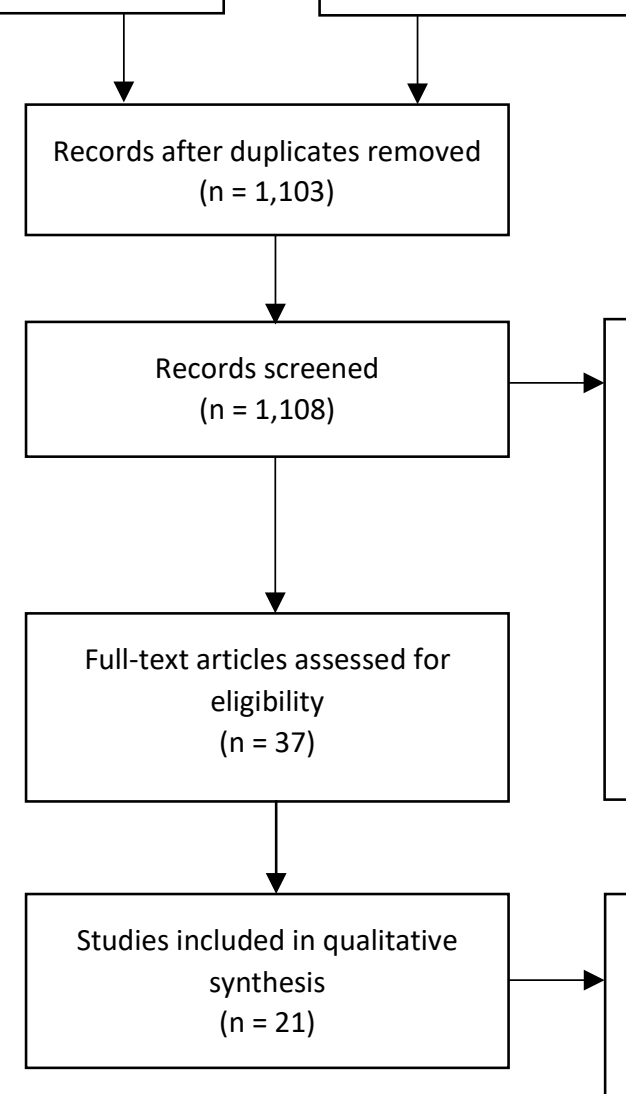

Full-text articles excluded, with reasons

$$
(n=16)
$$

- Discussion of barefoot rather than in-shoe pressure threshold $(n=8)$ 


\section{Results}

A total of 2,370 references were identified during the combined database searches, of which 1,267 were duplicates. 1,108 studies were screened (including 5 papers found through citations) based on an assessment of title and abstract of which 1,071 were discarded, including studies unconnected with diabetes or neuropathy, studies focused on the pressure sensing technology itself or pressure monitoring without any attempt to derive or apply in-shoe thresholds. Overall, 37 studies full text articles were reviewed from which 21 peer-reviewed research articles were selected for inclusion in accordance with the eligibility criteria (see Figure 1 above).

\subsection{Demographics}

All 21 studies were completed within the last 11 years, ranging from 2009 to 2019 including numbers of participants from 10 [33] to 171 [34, 42, 48]. Age, where provided for the whole cohort, varies between $57.0 \pm 9.0[35]-68.0 \pm 9.0$ [36], with the percentage of male participants ranging from $38.5 \%$ [36] - 89.0\% [37] (Table 1). A breakdown of type 1 and type 2 diabetes participants is available in 9 studies ranging between 28.6\% type 1 [38] to $41.0 \%$ [39] (Table 1). Duration of diabetes (reported in 12 studies) ranges between $12.6 \pm 7.6$ [36] - 22.0 \pm 15.9 [40].

\subsection{Current In-shoe Pressure Thresholds}

\subsection{1 $200 \mathrm{kPa}$ in-shoe pressure threshold}

Five in-shoe pressure thresholds were found and summarised (Table 2). Among these five thresholds, the most frequently applied was a 'mean peak pressure' of $200 \mathrm{kPa}$. This referred initially to the mean average of the maximum (peak) in-shoe plantar pressures at the previous ulcer location (region of interest) during 30 steps of walking [41] and later to the mean average of the maximum (peak) in-shoe plantar pressure at the specified regions of interest during a specified number of steps whilst walking (see regions of interest and no. of steps in Table 2). This $200 \mathrm{kPa}$ threshold was used primarily as a benchmark in the design and modification of footwear $(n=9)[34,35,39,40-44$, $50]$, insoles $(n=3)[36,45,46]$ and orthoses $(n=1)$ [47] but also in the analysis of equinus $(n=1)[22]$ and real-time modification of gait to reduce pressure ("biofeedback") $(n=2)[34,51]$. One study was unique in using a $450 \mathrm{kPa}$ barefoot pressure threshold in the initial selection of regions of interest for optimised insole designs later assessed using the $200 \mathrm{kPa}$ threshold [46].

\subsection{2. $25 \%$ baseline in-shoe pressure reduction target}

Some studies aimed to either reduce in-shoe pressure below $200 \mathrm{kPa}$ or by $25 \%(n=4)[34,44,39$ 40]. A footwear offloading target of $25 \%$ was chosen to represent a "significant, probably clinically relevant reduction in plantar pressure" [44]. In another study, only 16 of 50 previous ulcer locations had pressures above $200 \mathrm{kPa}$ [40] which may also have been a factor in developing this alternate $25 \%$ reduction target for footwear modification.

\subsubsection{0-80\% baseline in-shoe pressure reduction target}

An alternate in-shoe pressure reduction target of $40-80 \%$ of baseline at the area of highest peak plantar pressure has been used in biofeedback, the process of adjusting walking strategy/gait to reduce in-shoe pressures. The aim was to assess the feasibility of achieving this reduction for a minimum of seven steps out of each ten during three trials of walking in habitual footwear during 
both the learning phase and during ten-day follow-up $[38,51]$ or two week and six week follow-up [52]. This large threshold target range appears to be based on its potential achievability following a short review of studies of reductions within healthy volunteers, and to facilitate ease of learning [51].

\subsubsection{Low sustained in-shoe pressure threshold of 32-35 $\mathrm{mmHG}$}

A low sustained pressure in-shoe threshold of $32 \mathrm{mmHG}$ (millimetre of mercury, a pressure measure equivalent to $4.3 \mathrm{kPa}$ ) for a fifteen-minute period was suggested in Ferber et al. [33] based on the minimum pressure reported to cause bed sores. However, the study was limited to ten healthy participants, and intended simply to evaluate the SurroSense pressure monitoring insole against a gold standard. A subsequent RCT by Abbott et al. [37] evaluated a similar threshold of a sustained pressure exceeding $35 \mathrm{mmHG}$ in-shoe for $95 \%$ of the preceding 15 minutes against DFU outcomes in 58 participants with diabetes and neuropathy. Real-time biofeedback alerts suggested wearers should modify their stance or position, or rest.

\subsubsection{Risk, shoe size and foot region threshold matrix}

Giacomozzi proposed a matrix of thresholds depending on (1) the regions of the foot (toes, forefoot, midfoot or rearfoot); (2) the level of risk (level 0 = no neuropathy, history of ulceration or foot deformity, 1 = neuropathy but no deformity or history of ulceration; 2 = neuropathy and deformity but no history of ulceration or 3 = neuropathy, deformity and a history of ulceration) and (3) individual shoe size [49]. The suggested thresholds were based on reference in-shoe pressures obtained during walking in semi-rigid Terapes shoes with Pedar-X insoles for 20 healthy volunteers and 11 participants with diabetes and neuropathy.

\subsection{Outcomes}

The evidence of the effectiveness of these in-shoe pressure threshold is limited to just four studies $[34,37,41,48]$, consisting of two non-RCT cohort studies [41,48] and one RCT [34] relating to the $200 \mathrm{kPa}$ threshold and one RCT relating to the $35 \mathrm{mmHG}$ threshold [37]. The majority of these studies are non-RCT $(n=17)[22,33,35,36,38-46,48-51]$ concerned with the feasibility of various mechanisms of pressure reduction within the threshold target or as a benchmark for evaluating multiple interventions e.g. rival insoles or footwear designs. The only other RCT is concerned with pressure reduction outcomes in relation to cost and supply chain analysis [47] (Table 1).

This 200 kPa threshold is derived from Owings et al. (2009) [41] a small non-RCT where Pedar-X brand insole sensors were placed within prescription extra depth footwear with custom insoles to measure average in-shoe pressures of 49 people with diabetes and neuropathy who had been in remission from DFU for at least 90 days (average remission 3.6 years). The participants' mean inshoe pressure of 207 kPA whilst walking 30 steps, was rounded down to $200 \mathrm{kPa}$ to provide a target mean in-shoe peak pressure. Participants were typically wearing an extra-depth shoe with custommoulded insoles and a custom-moulded sandal for indoor use.

In a cohort study of 171 people with diabetes, neuropathy and a recently healed ulcer, wearing prescription footwear that reduced in-shoe pressure below this $200 \mathrm{kPa}$ threshold $(\mathrm{n}=171)$ for more than $80 \%$ of daylight hours led to a decreased risk of ulcer recurrence (odds ratio 0.43 [0.20-0.94]) [48]. 
The main evidence for this threshold comes from an RCT of 171 people with diabetes, neuropathy and a recently healed ulcer. The intervention group wore footwear which reduced pressures either below the $200 \mathrm{kPa}$ threshold or by $25 \%$ of baseline, which were evaluated at three monthly followups for up to 18 months and succeeded in decreased re-ulceration by $46 \%$ (odds ratio 0.38 [0.150.99], $p=0.045$ ) [34]. The $25 \%$ improvement in in-shoe pressure led to a non-significant relative risk reduction of $11 \%$ insufficient to significantly reduce ulcer recurrence risk [34] unless accompanied by sufficient adherence ( $80 \%$ of all steps taken in custom made footwear). The other studies (non-RCT) which used a target $25 \%$ pressure reduction did not evaluate the effect on ulceration rates, given their alternative aims of evaluating feasibility, optimisation and the number of rounds of modifications required to achieve this target [39-40,44].

Abbott et al. [37] is a RCT which evaluated a sustained pressure threshold exceeding $35 \mathrm{mmHG}$ inshoe for $95 \%$ of the preceding 15 minutes against DFU outcomes in 58 participants with diabetes and neuropathy. Real-time biofeedback alerts suggested wearers should modify their stance or position, or rest. A $71 \%$ reduction in the cumulative number of ulcer sites over total person days was achieved in the intervention group as compared with the control group (incident rate ratio $0.29,95 \%$ $\mathrm{Cl}, 0.09-0.93, \mathrm{p}=0.037$ ) in footwear worn on average 6.1 hours per day. Numbers of patients who ulcerated were 6 of 26 in the control group versus 4 of 32 in the intervention group.

The impact of a $40-80 \%$ reduction in baseline in-shoe pressure through biofeedback-based walking strategies on ulceration rates has yet to be evaluated $[38,51,52]$. To date, only one study has evaluated retention of these walking strategies beyond ten days, where only $50 \%$ achieved the required maximum pressure threshold in a small non-RCT cohort with just 11 participants [52].

Equally the suggested approach of a matrix of different thresholds providing thresholds for four regions (toes, forefoot, midfoot and rearfoot) based on both an individual's risk level and shoe size [49] has yet to be formally assessed against ulceration rates or days spent in remission. This protocol was derived from a validation session of only 11 participants with diabetes and neuropathy who were provided with modified prescription footwear and followed for only four months. The Terapes shoe with which reference data was also obtained has a very small heel and appears to be opentoed [49].

\subsection{Population within In-Shoe Pressure Threshold Studies}

Both the $200 \mathrm{kPa}$ and $35 \mathrm{mmHG}$ in-shoe pressure thresholds have been applied to prevent reulceration in those with diabetes, neuropathy and a history of ulceration $(n=11)[34,36,37,39-44$, $47,48]$. The $200 \mathrm{kPa}$ threshold was originally intended to prevent re-ulceration, but has since been applied to those with diabetes, neuropathy and no history of ulceration $(n=2)[35,45]$ or mixed groups of both $(n=3)[36,44,47]$, or with diabetes without neuropathy $(n=2)[35,50]$. Most studies aim to prevent re-ulceration in those with a "recently healed ulcer" $(n=8)[34,37,41-44,39-40,48]$ meaning an ulcer that healed within the 18 months prior to the study ( $n=6)[34,42-43,39-40,48]$ or which has been healed for more than 90 days $(n=1)$ with a mean of 3.6 years [41] or where the length of time since the ulcer healed is unspecified $(n=2)[44,37]$. 
In-shoe Pressure Threshold Study Demographics

\begin{tabular}{|c|c|c|c|c|c|c|c|c|c|c|}
\hline Paper, Year & Study Type & Size & Cohort & Recently Healed & DM1 & DM2 & Age & $M / F(\%)$ & Weight & $\begin{array}{l}\text { Duration } \\
\text { (Diabetes) }\end{array}$ \\
\hline Abbott, 2019 [37] & RCT (multicentre) & $\begin{array}{l}26 \\
32\end{array}$ & $\begin{array}{l}\mathrm{DN}+\mathrm{HU} \\
\mathrm{DN}+\mathrm{HU}\end{array}$ & $\begin{array}{l}\text { "recent history" } \\
\text { (not specified) }\end{array}$ & \multicolumn{2}{|c|}{58} & $\begin{array}{l}67.1 \pm 9.6 \\
59.1 \pm 8.5\end{array}$ & $\begin{array}{l}89.0 / 11.0 \\
88.0 / 12.0\end{array}$ & $\begin{array}{l}95.7 \pm 16.8 \\
102.1 \pm 18.9\end{array}$ & $\begin{array}{l}21.2 \pm 10.7 \\
22.2 \pm 14.3\end{array}$ \\
\hline Arts, 2012 [42] & Non-RCT. Cohort study & 171 & $\mathrm{DN}+\mathrm{HU}$ & $<18$ months & 49 & 122 & $62.8 \pm 10.2$ & 81.9 / 18.1 & -- & $\begin{array}{l}14.0 \pm 10.0- \\
27.5^{\sim}\end{array}$ \\
\hline Arts, 2015 [43] & $\begin{array}{l}\text { Non-RCT. Cohort study. } \\
\text { (From Bus } 2013 \text { below) }\end{array}$ & 85 & $\mathrm{DN}+\mathrm{HU}$ & $<18$ months & 28 & 57 & $62.6 \pm 10.2$ & $82.4 / 17.6$ & -- & $19.9 \pm 15.1$ \\
\hline Bus, $2011[44]$ & Noninterventional study & $\begin{array}{r}18 \\
5 \\
\end{array}$ & $\begin{array}{l}\mathrm{DN}+\mathrm{HU} \\
\mathrm{DN}\end{array}$ & Length of time not specified & 8 & 15 & $59.1 \pm 12.6$ & 73.9 / 26.1 & -- & $13.8 \pm 10.1$ \\
\hline Bus, 2013* [34] & $\begin{array}{l}\text { RCT. (With/without } \\
\text { pressure relief) }\end{array}$ & 171 & $\mathrm{DN}+\mathrm{HU}$ & $<18$ months & 49 & 122 & $63.3 \pm 10.2$ & 81.9 / 18.1 & -- & $19.9 \pm 15.1$ \\
\hline $\begin{array}{l}\text { Chapman, } 2014 \\
\text { [50] }\end{array}$ & $\begin{array}{l}\text { Non-RCT } \\
\text { Factorial design } \\
\text { (2-way ANOVA) }\end{array}$ & 87 & $\mathrm{D}$ & -- & -- & -- & -- & -- & -- & -- \\
\hline $\begin{array}{l}\text { De León Rodriguez, } \\
2013 \text { [38] }\end{array}$ & $\begin{array}{l}\text { Non-RCT. Cohort study. } \\
\text { (new walking strategy) }\end{array}$ & 21 & DN & -- & 6 & 15 & $57.8 \pm 2.9$ & $57.1 / 42.9$ & $85.8 \pm 3.6$ & $17.3 \pm 2.1$ \\
\hline Ferber, 2013 [33] & $\begin{array}{l}\text { Non-RCT. Prototype } \\
\text { sensor validation }\end{array}$ & 10 & CG & -- & -- & -- & $27.9 \pm 6.7$ & $70.0 / 30.0$ & $78.6 \pm 11.1$ & -- \\
\hline $\begin{array}{l}\text { Giacomozzi, } 2013 \\
* *[49]\end{array}$ & $\begin{array}{l}\text { Protocol + preliminary } \\
\text { validation }\end{array}$ & $\begin{array}{l}20 \\
11\end{array}$ & $\begin{array}{l}\text { CG } \\
\text { DN }\end{array}$ & -- & $\begin{array}{l}- \\
--\end{array}$ & $\begin{array}{l}- \\
--\end{array}$ & $\begin{array}{l}67.0 \pm 7.9 \\
70.2 \pm 6.7\end{array}$ & $\begin{array}{l}- \\
--\end{array}$ & $\begin{array}{l}75.0 \pm 8.1 \\
79.0 \pm 11.5\end{array}$ & $\begin{array}{l}- \\
--\end{array}$ \\
\hline Lin, 2013 [36] & $\begin{array}{l}\text { Non-RCT. Cohort study. } \\
\text { (plug removal insole) }\end{array}$ & $\begin{array}{l}26 \\
(3)\end{array}$ & $\begin{array}{l}\mathrm{DN} \\
\mathrm{DN}+\mathrm{HU}\end{array}$ & -- & 0 & 26 & $68.0 \pm 9.0$ & $38.5 / 61.5$ & $64.6 \pm 9.6$ & $12.6 \pm 7.6$ \\
\hline $\begin{array}{l}\text { Martinez-Santos, } \\
2019 \text { [45] }\end{array}$ & $\begin{array}{l}\text { Non-RCT. Cohort study ( } 9 \\
\text { insole variants) }\end{array}$ & 60 & DN & Excluded DN+HU & \multicolumn{2}{|c|}{60} & $67.3 \pm 13.0$ & 66.6 / 33.4 & -- & -- \\
\hline Owings, 2009 [41] & Non-RCT. Cohort study. & 49 & $\mathrm{DN}+\mathrm{HU}$ & $\begin{array}{l}>90 \text { days } \\
\text { Median } 3 \text { years since healing }\end{array}$ & -- & -- & $62.9 \pm 10.3$ & 77.6 / 22.4 & $88.1 \pm 17.5$ & \\
\hline Parker, 2019 [47] & $\begin{array}{l}\text { RCT (pressure reduction } \\
\text { outcomes + cost analysis) }\end{array}$ & $\begin{array}{r}57 \\
(23)\end{array}$ & $\begin{array}{l}\mathrm{DN} \\
\mathrm{DN}+\mathrm{HU}\end{array}$ & $>2$ years since $\mathrm{HU}$ & \multicolumn{2}{|c|}{57} & $\begin{array}{l}61.4 \pm 10.0 \\
66.3 \pm 10.5 \\
\end{array}$ & $\begin{array}{l}88.5 / 11.5 \\
84.6 / 15.4\end{array}$ & -- & -- \\
\hline Pataky, 2010 [51] & Non-RCT. Cohort study. & 13 & DN & -- & \multicolumn{2}{|c|}{13} & $60.8 \pm 12.3$ & $46.2 / 53.8$ & $82.9 \pm 14.1$ & $16.0 \pm 10.6$ \\
\hline Preece, 2017 [35] & $\begin{array}{l}\text { Non-RCT. Cohort study } \\
\text { (rocker sole design } \\
\text { evaluation). }\end{array}$ & $\begin{array}{r}102 \\
(17) \\
66 \\
\end{array}$ & $\begin{array}{l}\text { D } \\
\text { (DN) } \\
\text { CG }\end{array}$ & Excluded $\mathrm{DN}+\mathrm{HU}$ & \multicolumn{2}{|c|}{102} & $57.0 \pm 9.0$ & $51.0 / 49.0$ & $87.0 \pm 18.0$ & -- \\
\hline Searle, 2018 [22] & $\begin{array}{l}\text { Non-RCT. Cohort study } \\
\text { (equinus within diabetes) }\end{array}$ & $\begin{array}{l}71 \\
65\end{array}$ & $\begin{array}{l}\mathrm{D} \\
\mathrm{DN}\end{array}$ & -- & -- & -- & -- & -- & -- & -- \\
\hline
\end{tabular}




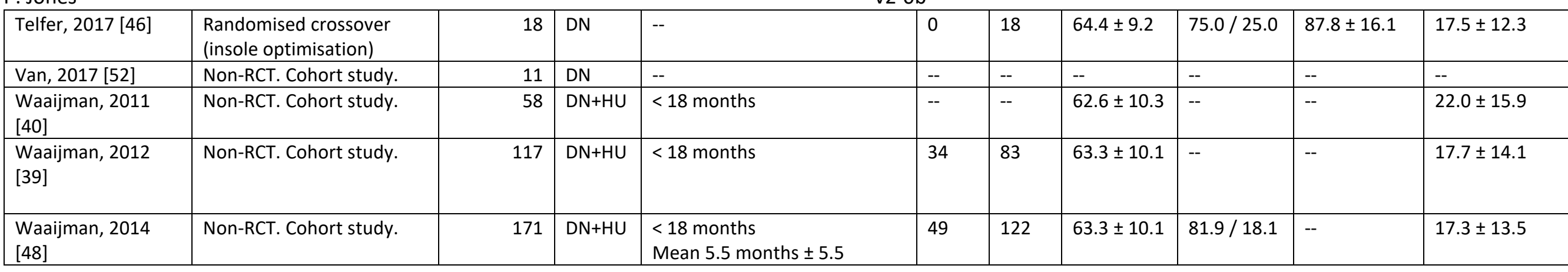

Key

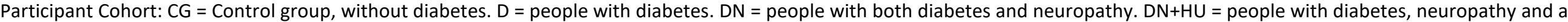
history of ulceration, usually a recently healed plantar foot ulcer. Brackets indicate a subgroup of the main cohort included within the total.

All demographics: mean \pm standard deviation unless $\sim$ indicating interquartile range. -- means data unavailable.

*Numbers with DM1/2 calculated from percentages in Table 1, p4111. Weighted mean calculation performed to obtain age mean and standard deviation.

** DNU implied from participants being 'high risk', having neuropathy and prescription footwear. 


\begin{tabular}{|c|c|c|c|c|c|c|c|c|c|}
\hline Paper, Year & Threshold Criteria & $\begin{array}{l}\text { Region of } \\
\text { Interest }\end{array}$ & $\begin{array}{l}\text { Sensor } \\
\text { Type }\end{array}$ & $\begin{array}{l}\text { No. Sub } \\
\text { Regions }\end{array}$ & $\begin{array}{l}\text { No. } \\
\text { Steps }\end{array}$ & $\begin{array}{l}\text { Footwear } \\
\text { Type }\end{array}$ & Pace & $\begin{array}{l}\text { Study } \\
\text { Duration }\end{array}$ & Purpose \\
\hline Abbott, 2019 [37] & $\begin{array}{l}\text { 95-100\% readings over past } 15 \text { mins } \geq 35 \mathrm{mmHG} * \\
\text { trigger audio-visual and vibratory alerts to offload }\end{array}$ & Entire foot & $\begin{array}{l}\text { Surro } \\
\text { Sense }\end{array}$ & $\begin{array}{c}8 \\
\text { sensors }\end{array}$ & $\mathrm{N} / \mathrm{A}$ & $\begin{array}{l}\text { Prescription } \\
\text { (Customised) }\end{array}$ & $\begin{array}{l}\text { Free } \\
\text { Living }\end{array}$ & $\begin{array}{l}18 \\
\text { months }\end{array}$ & Biofeedback \\
\hline Arts, $2012[42]$ & $\begin{array}{l}\text { Offloading target of in-shoe pressure }<200 \mathrm{kPa} \text { at previous } \\
\text { ulcer location and up to two other locations of highest } \\
\text { barefoot pressure attributable to deformity }\end{array}$ & Entire foot & $\begin{array}{c}\text { Pedar } \\
\quad \mathrm{X}\end{array}$ & $\begin{array}{c}10 \\
\text { regions }\end{array}$ & $\begin{array}{l}12 \\
\text { steps } \\
\text { per ft }\end{array}$ & $\begin{array}{l}\text { Fully }^{1} / \text { Semi }^{2} \\
\text { Custom } \\
85.4 \% / 14.6 \% \\
\end{array}$ & $\begin{array}{l}\text { Self - } \\
\text { Select } \\
1 \mathrm{~m} / \mathrm{s}\end{array}$ & $\begin{array}{l}\text { Single } \\
\text { session }\end{array}$ & $\begin{array}{l}\text { Footwear } \\
\text { Offloading } \\
\text { Deformity }\end{array}$ \\
\hline Arts, 2015 [43] & $\begin{array}{l}\text { Footwear modified at any location distal to the heel } \geq 200 \\
k P a \text { including previous ulcer location or site of deformity and } \\
\text { up to two other locations }\end{array}$ & $\begin{array}{l}\text { Forefoot } \\
+ \text { midfoot }\end{array}$ & $\begin{array}{l}\text { Pedar } \\
\quad \mathrm{X}\end{array}$ & $\begin{array}{c}8 \\
\text { regions }\end{array}$ & $\begin{array}{l}12 \\
\text { steps } \\
\text { per } \mathrm{ft}\end{array}$ & $\begin{array}{l}\text { Fully Custom }{ }^{1} \\
100 \%\end{array}$ & $\begin{array}{l}\text { Self - } \\
\text { Select }\end{array}$ & $\begin{array}{l}15 \\
\text { months }\end{array}$ & $\begin{array}{l}\text { Evaluate } \\
\text { Footwear } \\
\text { Modification }\end{array}$ \\
\hline Bus, $2011[44]$ & $\begin{array}{l}\text { Footwear modified where previous ulcer location or location } \\
\text { of deformity or pre-ulcerative signs }>200 \mathrm{kPa} \text { or where two } \\
\text { other peak pressure locations }>300 \mathrm{kPa} \text {. } \\
\text { Modification successful if }<200 \mathrm{kPa} \text { or in-shoe pressure } \\
\text { reduced by } 25 \%\end{array}$ & Entire foot & $\begin{array}{c}\text { Pedar } \\
\quad \mathrm{X}\end{array}$ & $\begin{array}{c}10 \\
\text { regions }\end{array}$ & $\begin{array}{l}15 \\
\text { steps }\end{array}$ & $\begin{array}{l}\text { Fully Custom }^{1} \\
100 \%\end{array}$ & $\begin{array}{l}\text { Self - } \\
\text { Select }\end{array}$ & $\begin{array}{l}\text { Single } \\
\text { session } \\
\text { (multi } \\
\text { tests) }\end{array}$ & $\begin{array}{l}\text { Evaluate } \\
\text { Footwear } \\
\text { Modification }\end{array}$ \\
\hline Bus, $2013[34]$ & $\begin{array}{l}\text { Footwear designed to reduce in-shoe pressure at previous } \\
\text { ulcer location or two other locations }<200 \mathrm{kPa} \text { or reduction } \\
\text { by } 25 \% \text { baseline. Effect on ulceration with and without }>85 \% \\
\text { adherence }\end{array}$ & $\begin{array}{l}\text { Forefoot } \\
+ \text { midfoot }\end{array}$ & $\begin{array}{l}\text { Pedar } \\
\quad X\end{array}$ & -- & $\begin{array}{l}15 \\
\text { steps }\end{array}$ & $\begin{array}{l}\text { Fully }^{1} / \text { Semi }^{2} \\
\text { Custom } \\
85.4 \% / 14.6 \%\end{array}$ & $\begin{array}{l}\text { Self - } \\
\text { Select }\end{array}$ & $\begin{array}{l}18 \\
\text { months }\end{array}$ & $\begin{array}{l}\text { Footwear \& } \\
\text { ulceration } \\
\text { study }\end{array}$ \\
\hline $\begin{array}{l}\text { Chapman, } 2014 \\
{[50]}\end{array}$ & Threshold of $200 \mathrm{kPa}$ used to evaluate rocker shoes & -- & -- & -- & -- & $\begin{array}{l}\text { Fully Custom } \\
\text { (Rocker shoe) }\end{array}$ & $1 \mathrm{~m} / \mathrm{s}$ & $\begin{array}{l}\text { Single } \\
\text { session }\end{array}$ & $\begin{array}{l}\text { Evaluate } \\
\text { Footwear }\end{array}$ \\
\hline $\begin{array}{l}\text { De León Rodriguez, } \\
2013 \text { [38] }\end{array}$ & $\begin{array}{l}\text { Developing a pressure-reduction walking strategy based on } \\
40-80 \% \text { baseline in-shoe pressures for } 70 \% \text { of totality of steps }\end{array}$ & Entire foot & $\begin{array}{c}\text { Pedar } \\
\mathrm{X}\end{array}$ & $\begin{array}{c}11 \\
\text { regions }\end{array}$ & $\begin{array}{l}10 \\
\text { steps }\end{array}$ & $\begin{array}{l}\text { Everyday } \\
\text { shoes }\end{array}$ & $\mathrm{N} / \mathrm{A}$ & 10 days & Biofeedback \\
\hline Ferber 2013 [33] & $\begin{array}{l}\text { Comparison of SurroSense device sensors with Pedar-X. Bed } \\
\text { sore pressure threshold of } \geq 32 \mathrm{mmHG} \text { suggested for use in } \\
\text { diabetic foot ulceration without detailed discussion }\end{array}$ & Entire foot & $\begin{array}{l}\text { Surro } \\
\text { Sense }\end{array}$ & $\begin{array}{c}8 \\
\text { sensors }\end{array}$ & N/A & Retail shoes & $\begin{array}{l}\text { Self - } \\
\text { Select }\end{array}$ & $\begin{array}{l}\text { Single } \\
\text { session }\end{array}$ & $\begin{array}{l}\text { Sensor Test } \\
\text { Biofeedback }\end{array}$ \\
\hline $\begin{array}{l}\text { Giacomozzi } 2013 \\
\text { [49] }\end{array}$ & $\begin{array}{l}\text { Matrix of target pressures for four regions (toes, forefoot, } \\
\text { midfoot, rearfoot) stratified by risk level and shoe size } \\
\text { proposed to minimise ulceration }\end{array}$ & Entire foot & $\begin{array}{l}\text { Pedar } \\
\quad \mathrm{X}\end{array}$ & $\begin{array}{c}4 \\
\text { regions }\end{array}$ & $\begin{array}{l}20 \\
\text { steps } \\
\text { per } \mathrm{ft}\end{array}$ & $\begin{array}{l}\text { Post-surgery } \\
\text { semi-rigid } \\
\text { (12 mths old })\end{array}$ & $\begin{array}{l}\text { Self - } \\
\text { Select }\end{array}$ & $\begin{array}{l}4 \\
\text { months }\end{array}$ & $\begin{array}{l}\text { Suggest } \\
\text { Threshold }\end{array}$ \\
\hline Lin 2013 [36] & $\begin{array}{l}\text { Site of highest mean forefoot pressure is region of interest. } \\
\text { Target of } 200 \mathrm{kPa} \text { used to evaluate insole }\end{array}$ & Forefoot & $\begin{array}{c}\text { Pedar } \\
X\end{array}$ & $\begin{array}{c}5 \\
\text { regions }\end{array}$ & $\begin{array}{l}30 \\
\text { steps }\end{array}$ & $\begin{array}{l}\text { Extra depth } \\
\text { shoes }\end{array}$ & $\begin{array}{l}\text { Self - } \\
\text { Select }\end{array}$ & $\begin{array}{l}\text { Single } \\
\text { session }\end{array}$ & $\begin{array}{l}\text { Evaluate } \\
\text { Insole }\end{array}$ \\
\hline $\begin{array}{l}\text { Martinez-Santos } \\
2019[45]\end{array}$ & $\begin{array}{l}\text { Target of mean forefoot pressure }<200 \mathrm{kPa} \text { used to evaluate } \\
\text { nine alternative insole designs }\end{array}$ & Forefoot & $\begin{array}{c}\text { Pedar } \\
X\end{array}$ & $\begin{array}{c}5 \\
\text { regions }\end{array}$ & $\begin{array}{l}20 \\
\text { steps }\end{array}$ & $\begin{array}{l}\text { Retail shoes } \\
\text { (Standard) }\end{array}$ & $\begin{array}{l}\text { Self - } \\
\text { Select }\end{array}$ & $\begin{array}{l}\text { Single } \\
\text { session }\end{array}$ & $\begin{array}{l}\text { Evaluate } \\
\text { Insoles }\end{array}$ \\
\hline
\end{tabular}




\begin{tabular}{|c|c|c|c|c|c|c|c|c|c|}
\hline Paper, Year & Threshold Criteria & Location & $\begin{array}{l}\text { Sensor } \\
\text { Type }\end{array}$ & $\begin{array}{l}\text { No. } \\
\text { Regions }\end{array}$ & $\begin{array}{l}\text { No. } \\
\text { Steps }\end{array}$ & $\begin{array}{l}\text { Footwear } \\
\text { Type }\end{array}$ & Pace & $\begin{array}{l}\text { Study } \\
\text { Duration }\end{array}$ & Purpose \\
\hline Owings 2009 [41] & $200 \mathrm{kPa}$ target established to prevent re-ulceration & $\begin{array}{l}\text { Prior ulcer } \\
\text { site } \\
\text { (Hallux }+ \\
\text { MTHs) }\end{array}$ & Pedar & $\begin{array}{c}3 \\
\text { regions }\end{array}$ & $\begin{array}{l}30 \\
\text { steps }\end{array}$ & $\begin{array}{l}\text { Prescription } \\
\text { Extra depth } \\
\text { shoes }\end{array}$ & $\begin{array}{l}\text { Self - } \\
\text { Select }\end{array}$ & $\begin{array}{l}\text { Single } \\
\text { session }\end{array}$ & $\begin{array}{l}\text { Suggest } \\
\text { Threshold }\end{array}$ \\
\hline Parker, 2019 [47] & $\begin{array}{l}\text { Evaluation of orthoses and secondary aim of reducing } \\
\text { pressures at site of highest pressure and up to three regions } \\
\text { at risk which are }>200 \mathrm{kPa}\end{array}$ & $\begin{array}{l}\text { Hallux + } \\
\text { MTHs }\end{array}$ & Pedar & $\begin{array}{c}3 \\
\text { regions }\end{array}$ & $\begin{array}{l}30 \\
\text { steps } \\
\text { per } \mathrm{ft}\end{array}$ & $\begin{array}{l}\text { Everyday } \\
\text { shoes }\end{array}$ & $\begin{array}{l}\text { Self - } \\
\text { Select }\end{array}$ & $\begin{array}{l}6 \\
\text { months }\end{array}$ & $\begin{array}{l}\text { Evaluate } \\
\text { Orthoses }\end{array}$ \\
\hline Pataky, $2010[51]$ & $\begin{array}{l}\text { Minimum of seven steps reduced to within } 40-80 \% \text { baseline } \\
\text { pressure at highest pressure regions through biofeedback / } \\
\text { walking strategies }\end{array}$ & $\begin{array}{l}\text { Highest } \\
\text { pressure } \\
\text { region }\end{array}$ & Pedar & -- & $\begin{array}{l}10 \\
\text { steps }\end{array}$ & $\begin{array}{l}\text { Everyday } \\
\text { shoes }\end{array}$ & $\begin{array}{l}\text { Self - } \\
\text { Select }\end{array}$ & $\begin{array}{l}\text { Baseline, } \\
@ 1,5, \\
10 \text { days }\end{array}$ & Biofeedback \\
\hline Preece, 2017 [35] & $\begin{array}{l}\text { Target of }<200 \mathrm{kPa} \text { used to evaluate footwear intended to } \\
\text { prevent first-ulceration }\end{array}$ & $\begin{array}{l}\text { Hallux, } \\
\text { MTHs, } \\
\text { MTP }\end{array}$ & $\begin{array}{l}\text { Pedar } \\
\quad \mathrm{X}\end{array}$ & $\begin{array}{l}3 \\
\text { regions }\end{array}$ & $\begin{array}{l}25 \\
\text { steps } \\
\text { per } \mathrm{ft}\end{array}$ & $\begin{array}{l}\text { Group } \\
\text { optimised } \\
\text { shoes }\end{array}$ & $\begin{array}{l}\text { Pace } \\
1 \mathrm{~m} / \mathrm{s}\end{array}$ & $\begin{array}{l}\text { Single } \\
\text { session }\end{array}$ & $\begin{array}{l}\text { Evaluate } \\
\text { Footwear }\end{array}$ \\
\hline Searle, 2018 [22] & $\begin{array}{l}\text { Analysis of relationship between equinus and high pressure } \\
\text { defined as } \geq 200 \mathrm{kPa} \text { and low pressure }<200 \mathrm{kPa}\end{array}$ & Entire foot & $\begin{array}{l}\text { Pedar } \\
\quad \mathrm{X}\end{array}$ & $\begin{array}{l}5 \\
\text { regions }\end{array}$ & $\begin{array}{l}12 \\
\text { steps } \\
\text { per ft* }\end{array}$ & $\begin{array}{l}\text { Everyday } \\
\text { shoes } \\
\text { (trainer) }\end{array}$ & $\begin{array}{l}\text { Self - } \\
\text { Select }\end{array}$ & $\begin{array}{l}\text { Single } \\
\text { session }\end{array}$ & $\begin{array}{l}\text { Evaluate } \\
\text { equinus \& } \\
\text { pressure }\end{array}$ \\
\hline Telfer, 2017 [46] & $\begin{array}{l}\text { Hybrid: Owings barefoot pressure threshold of } 450 \mathrm{kPa} \text { used } \\
\text { to select regions of interest } \\
\text { Owings } 200 \mathrm{kPa} \text { target used to assess insoles and determine } \\
\text { how much insole material to remove }\end{array}$ & MTHs & $\begin{array}{l}\text { Pedar } \\
\quad \mathrm{X}\end{array}$ & $\begin{array}{l}3 \\
\text { regions }\end{array}$ & $\begin{array}{l}12 \\
\text { steps } \\
\text { per } \mathrm{ft}\end{array}$ & $\begin{array}{l}\text { Extra depth } \\
\text { shoes }\end{array}$ & $\begin{array}{l}\text { Self - } \\
\text { Select }\end{array}$ & $\begin{array}{l}\text { Single } \\
\text { session }\end{array}$ & $\begin{array}{l}\text { Evaluate } \\
\text { Insoles }\end{array}$ \\
\hline Van, 2017 [52] & $\begin{array}{l}40-80 \% \text { reduction in baseline in-shoe peak pressures for } 70 \% \\
\text { of the totality of steps }\end{array}$ & Entire foot & Feetme & -- & $\begin{array}{l}20 \\
\text { steps }\end{array}$ & -- & $\begin{array}{l}\text { Self - } \\
\text { Select }\end{array}$ & $\begin{array}{l}\text { Baseline, } \\
\text { @ } 2,6 \\
\text { weeks }\end{array}$ & Biofeedback \\
\hline Waaijman, $2011[40]$ & $\begin{array}{l}\text { Footwear modification regions of interest based on previous } \\
\text { ulcer location and highest two pressure areas }>200 \mathrm{kPa} \\
\text { Target: }<200 \mathrm{kPa} \text { or } 25 \% \text { reduction in pressure }\end{array}$ & -- & $\begin{array}{l}\text { Pedar } \\
\quad \mathrm{X}\end{array}$ & -- & -- & $\begin{array}{l}\text { Fully }^{1} / \text { Semi }^{2} \\
\text { Custom }\end{array}$ & -- & -- & $\begin{array}{l}\text { Evaluate } \\
\text { Footwear } \\
\text { Modification }\end{array}$ \\
\hline Waaijman, 2012 [39] & $\begin{array}{l}\text { Footwear modification regions of interest based on previous } \\
\text { ulcer location and highest two pressure areas }>200 \mathrm{kPa} \\
\text { (midfoot or forefoot) } \\
\text { Target: }<200 \mathrm{kPa} \text { or } 25 \% \text { reduction in pressure }\end{array}$ & Entire foot & $\begin{array}{l}\text { Pedar } \\
\quad \mathrm{X}\end{array}$ & $\begin{array}{l}10 \\
\text { regions }\end{array}$ & $\begin{array}{l}20 \\
\text { steps }\end{array}$ & $\begin{array}{l}\text { Fully }{ }^{1} / \text { Semi }^{2} \\
\text { Custom } \\
81.2 \% / 18.8 \\
\%\end{array}$ & $\begin{array}{l}\text { Self - } \\
\text { Select }\end{array}$ & $\begin{array}{l}12 \\
\text { months }\end{array}$ & $\begin{array}{l}\text { Evaluate } \\
\text { Footwear } \\
\text { Modification }\end{array}$ \\
\hline Waaijman, 2014 [48] & $\begin{array}{l}\text { In-shoe pressure monitored in custom-made shoes every } 3 \\
\text { months for } 18 \text { months or until ulceration. } \\
\text { Previous ulcer location or plantar area }>200 \mathrm{kPa}\end{array}$ & Entire foot & $\begin{array}{l}\text { Pedar } \\
\quad \mathrm{X}\end{array}$ & $\begin{array}{l}10 \\
\text { regions }\end{array}$ & $\begin{array}{l}12 \\
\text { steps } \\
\text { per } \mathrm{ft}\end{array}$ & $\begin{array}{l}\text { Fully }^{1} / \text { Semi }^{2} \\
\text { Custom }\end{array}$ & $\begin{array}{l}\text { Self - } \\
\text { Select }\end{array}$ & $\begin{array}{l}18 \\
\text { months }\end{array}$ & $\begin{array}{l}\text { Pressure \& } \\
\text { Ulceration } \\
\text { Study }\end{array}$ \\
\hline
\end{tabular}




\section{Key}

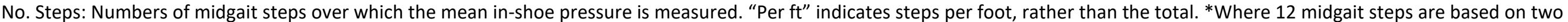
walking trials, each consisting of six mid-gait steps.

Footwear: ${ }^{1}$ Both custom insoles and custom-made shoes; ${ }^{2}$ Custom insoles and extra depth shoes. -- indicates no information provided. 
The most common approach for testing loss of protective sensation is a $10 \mathrm{~g}$ monofilament $(n=11)$ applied to the hallux $(n=8)$, first and fifth metatarsals $(n=8)$ although there was considerable variation both in number and location of sites tested, and the combination of tests applied (see Supplemental Table 4 for details) rendering meta-analysis impossible. There was no in-depth analysis on the impact of either severity of neuropathy or peripheral vascular disease in relation to ulceration within these studies.

\subsection{Sensor Types and Region of Interest}

Most of the studies used Pedar-X insoles to measure laboratory-based in-shoe peak pressures $(n=17)$ whereas the SurroSense insole system provided alerts which flagged sustained low pressures in free living $(n=2)[33,37]$. The SurroSense insole measures in-shoe pressure using 8 sensors (five located at the forefoot, one at the midfoot and two at the heel) whereas the Pedar-X system employs 99 sensors across the plantar region which can be sub-divided into regions of interest (ROI). A FeetMe insole to measure max peak pressure reduction was also used in a biofeedback walking strategy study although details of the number and location of sensors is not provided [52]. The $200 \mathrm{kPa}$ threshold was intended to prevent forefoot re-ulceration (primarily hallux and metatarsals regions) and has since been applied to the entire plantar region $(n=4)[22,39,42,44]$, forefoot and midfoot $(n=2)[34,43]$, forefoot $(n=2)[36,45]$, hallux, metatarsals and metatarsophalangeal joint [35], hallux and metatarsals $(n=1)$ [47] and metatarsals $(n=1)$ [46].When reporting pressures by region, subdivision of the plantar region varies from 4 regions [49] to 11 (Table 1) [38]. Within these $200 \mathrm{kPa}$ threshold studies, the maximum number of target regions for pressure reduction varied between 1 (usually to test mean in-shoe pressure to evaluate footwear, insoles or orthoses) [36, 45, 49] - 4 [47] where the most common approach targeted the previous ulcer location (see Supplemental Table 2) and the two highest pressure locations $(n=5)[39-40,42,44]$ (Table 2).

\subsection{Number of steps required to calculate mean in-shoe pressure}

The target mean in-shoe peak pressure of $200 \mathrm{kPa}$ developed in Owings [41] was based on approximately 30 steps i.e. the mean average peak pressure detected at the previous ulcer location during those 30 steps. Similarly, mean peak pressure is applied in other studies [22, 34-36, 39-45, 47, $48,50]$. However, in studies which subsequently applied this threshold this varies between 12 steps $[42,43]$ and 30 steps per foot [47].

\subsection{Length of study and frequency of pressure measurement}

The length of these studies and therefore the number of times in-shoe pressure is measured also varied: in $200 \mathrm{kPa}$ studies to evaluate footwear, insole and orthoses designs, these ranged from a single measurement to evaluation over a six month period [47], or at three month intervals when evaluating footwear modifications from 12 [39] to 18 months $(n=2)$ [34, 48]. Biofeedback studies ranged from 10 days $(200 \mathrm{kPa})[38,51]$ with three sets of laboratory in-shoe pressure measurements to 18 months (35 $\mathrm{mmHG}$ ) in free-living [37].

\subsection{Footwear worn during in-shoe pressure measurement}

Footwear customised to the individual were often used ( $n=10[34,37,39-44,48,50])$, or off-the- 
shelf retail or everyday footwear $(n=6)[22,33,38,45,47,51]$, and standardised footwear in the form of either extra depth $(n=2)[36,46]$, semi-rigid footwear intended for post-surgery use [49] or group-optimised footwear [35] (Table 2) or unspecified [52]. Studies measuring in-shoe pressures within customised footwear either involved all participants wearing prescription $(n=1)$ [37] or 'fullycustomised' footwear (i.e. both a custom insole and custom shoe) $(n=2)$ [43-44] or a mixture of fully and semi-customised footwear consisting of extra-depth shoes with a custom insole $(n=7)[34,39$, $40-42,48,50]$ (Table 2). Only 4 studies explicitly mention standardisation of socks (Supplemental Table 3) [36, 39, 42-43].

\section{Discussion}

The relative contribution of peak pressure versus sustained low pressures within shoes to diabetesrelated ulceration remains unknown. We suspect that increased risk arises through a combination of both forms of in-shoe pressure combined with step count, type and intensity of physical activity or changes in lifestyle but RCT-based evidence for this hypothesis remains scarce. Technological barriers have prevented us thus far from capturing the complete picture: physical activity, peak and sustained low in-shoe pressures during in the run-up prior to ulceration. We need this to answer fundamental questions like 'Are dynamic peak pressures during walking more damaging to diabetic foot skin and tissue than sustained static pressures during standing or driving'? Suggested biofeedback interventions have developed around both a reduction in peak and sustained low pressures but have yet to be compared or combined within randomised controlled trials which assess ulceration rates and days spent in remission.

The evidence for both these in-shoe pressure thresholds is itself limited to one RCT for the 35mmHG sustained pressure threshold $(n=58)$ [37] and one RCT $(n=171)$ [34], and two cohort studies $(n=49$ [41], $n=171$ [48]) for the $200 \mathrm{kPa}$ peak pressure threshold. These studies are all focused on the prevention of re-ulceration. A separate threshold for first ulceration for those with diabetes and neuropathy or PVD has yet to be evaluated ( 7 studies focus on recently healed ulcers - Table 1 ). The remaining suggested thresholds of $40-80 \%$ reduction in baseline pressure $[38,51]$ and shoe size and foot region threshold matrix [49] have yet to be assessed against ulceration rates or days spent in remission.

Our review has revealed considerable variation in the number of regions analysed, the subdivision of those forefoot or plantar region analysed, or the number of steps over which the 200 KPA threshold is assessed - all required some standardisation for facilitate ready comparison. Arts and Bus recommend a minimum number of twelve steps per foot (24 in total) [53] necessary for statistically reliable data. In research analysing in-shoe pressures and ulceration outcomes, assessment of footwear fit is another important element (the International Working Group on Diabetic Foot [54] guidelines recommended a 1-2 cm toe gap) given its potential impact on in-shoe pressure [55].

Obstacles to meta-analysis have included varying methods of assessing loss of protective sensation, and a lack of sub-group analysis around severity of neuropathy and peripheral vascular disease. A possible approach might be to employ a system of ranges either around neuropathy disability score as in Caselli [56] : 0 as "absent", 1-5 as "mild", 6-16 as "moderate" and 17+ as "severe" or alternatively based around VPT as in Mondal et al. [57] consisting of 25-35V as "mild"; 36-50 as "moderate" and > 50V as "severe". Similarly with PVD, Society for Vascular Surgery WIfI (wound, ischemia, foot infection) classification might be employed [58], ankle brachial index of $\geq 0.80$ as 
"Grade 0", $0.6-0.79$ as "Grade 1", $0.4-0.59$ as "Grade 2", and $\leq 0.39$ as "Grade 3" or a similar classification system [59]. Together with past ulcer history, this may assist us to develop separate inshoe pressure thresholds for those with a history of ulceration versus recommended thresholds for those at risk of a first ulcer, stratified by these risk factors.

A range of commercial in-shoe pressure systems exist each with a differing number of sensors, sensor mechanism, measurement range, insole thickness and sampling rate [60]. This means that measurements taken with one system may not readily facilitate comparison with another as each works differently. For instance, in Owings [41] Pliance sensors were also used, taped to the previous ulcer location, and produced values which were larger than those produced using the Pedar system which was attributed to a smaller sensor size [41]. The relative merits of each system and their performance are beyond the scope of this review [60].

A limitation of this review is that the data extraction, assessment and extraction were performed by one reviewer (PJ). Our review was also limited to the use of in-shoe pressure thresholds in the prevention of diabetes-related foot ulceration - another useful review might address their use in assisting active ulcer healing [61] or Charcot remission. A previous narrative analysis [29] covered both barefoot and in-shoe pressure analysis but we attempted a more comprehensive treatment of specific in-shoe pressure thresholds through a formal systematic review to reveal both the multiple ways in which thresholds are currently employed and the wide variation in their usage.

This systematic review is focused on in-shoe plantar pressure in isolation, but pressure is just one aspect of foot microclimate monitoring that potentially includes temperature and humidity [62] not to mention growing research around wearables such as accelerometers which can measure physical activity type and intensity. In-shoe pressure thresholds may become part of multi-factorial prognostic models.

\section{Conclusion}

Five in-shoe pressure thresholds were identified, which are employed to reduce the risk of diabetesrelated foot ulceration: a mean peak pressure threshold of $200 \mathrm{kPa}$ used in conjunction with a $25 \%$ baseline reduction target; a sustained pressure threshold of $35 \mathrm{mmHG}$, a threshold matrix based on risk, shoe size and foot region, and a $40-80 \%$ baseline pressure reduction target. The effectiveness of the latter two thresholds have not been assessed yet and the evidence for the effectiveness of the other in-shoe pressure thresholds is limited, based only on two RCTs and two cohort studies. The contribution of peak in-shoe pressures to foot ulceration as opposed to sustained low pressures remains as is their potential impact on ulceration rates and days spent in remission if they were combined. Divergence in methodological approaches around footwear, insole and socks, region of interest, loss of protective sensation preclude meta-analysis and standardisation is needed. Further research is needed as described below.

\section{Summary Recommendations}

Further randomised controlled trials are needed to assess the efficacy of footwear interventions based around in-shoe pressure threshold on ulceration rates and days spent in remission which:

(i) Track both step count and physical activity type and intensity whilst wearing pressure minimising 
footwear (necessitating pedometer, accelerometer and adherence data capture)

(ii) In-shoe peak pressure measurements are recorded every 3-months (based on a standardised 12 steps) and the number of days elapsed between measurement and any subsequent ulceration event recorded

(iii) Sustained low pressures ( $35 \mathrm{mmHg}$ ) during daily living should also be monitored to provide useful data regarding the impact of both thresholds upon ulceration

(iv) Standardise socks, insole and footwear (prescription extra depth)

(v) Research should prioritise people with diabetes at high risk of ulceration i.e. category 2 of the risk classification system of the International Working Group on the Diabetic Foot (IWGDF) with either a diagnosis of neuropathy or peripheral artery disease (PAD) and a history of ulceration. We suggest standardising loss of protective sensation testing to a $10 \mathrm{~g}$ monofilament applied to the hallux, first and fifth metatarsals and VPT $\geq 25 \mathrm{~V}$ (the most common approach found in these studies - see Supplemental Table 4). Severity of neuropathy and PVD should also be graded by VPT and ankle brachial index response.

The heterogeneity of current measures precludes us from conducting meta-analysis. Our review has revealed the importance of consistent application and minimal methodological variation in studies applying in-shoe pressure thresholds. Standardised methods enable us to conduct meta-analyses and readily draw comparisons through which we may build on past research to derive locationspecific thresholds or formulate multi-factorial pressure thresholds for use in diabetes-related ulcer prevention.

\section{CONFLICT OF INTERESTS}

The authors declare that they have no conflict interests.

\section{AUTHORS' CONTRIBUTION}

PJ conceived and designed the study, performed the literature search, assessed the literature, extracted data and drew conclusions. PJ and DW wrote the manuscript. DW, MJD, KK, RB, and PC critically reviewed and edited the manuscript. All authors have read and approved the final manuscript.

\section{ORCID}

Petra Jones https://orcid.org/0000-0002-9340-9168

David Webb https://orcid.org/0000-0002-3932-3339

\section{ACKNOWLEDGEMENTS}

The authors would like to thank UHL Libraries and Information Services for their ongoing and invaluable assistance and the ongoing support of NIHR Leicester Biomedical Research Centre with this area of research. 


\section{REFERENCES}

1. International Diabetes Federation (IDF) Diabetes Atlas Ninth Edition 2019 https://www.idf.org/aboutdiabetes/what-is-diabetes/facts-figures.html [Accessed 5 May 2020].

2. American Diabetes Association Consensus Development Conference on Diabetic Foot Wound Care: 7-8 April 1999, Boston, Massachusetts. Diabetes Care. 1999; 22:1354-1360.

3. Boulton AJM. The diabetic foot. Medicine 2015; 43:33-37. Available from https://www.medicinejournal.co.uk/article/S1357-3039(14)00296-5/pdf (Accessed 27/07/20).

4. Rice JB, Desai U, Cummings AK, Birnbaum HG, Skornicki M, Parsons NB, Burden of diabetic foot ulcers for medicare and private insurers 2014. Diabetes Care 37(3):651-658. https://doi.org/10.2337/dc13-2176.

5. Kerr M (2017) Improving footcare for people with diabetes and saving money: an economic study in England. Available from http://www.londonscn.nhs.uk/wp-content/uploads/2017/04/dia-footcare-mtg-kerr-27042017.pdf. (Accessed 30/09/20).

6. Lynar SA, Robinson $\mathrm{CH}$, Boutlis CS, Commons RJ, Risk factors for mortality in patients with diabetic foot infections: A prospective cohort study. Internal Medicine Journal 2019; 49:867-873. https://doi.org/10.1111/imj.14184.

7. Vadiveloo T, Jeffcoate W, Donnan PT, Colhoun HC, McGurnaghan S, Wild S, McCrimmon R, Leese GP, Amputation-free survival in 17,353 people at high risk for foot ulceration in diabetes: A national observational study. Diabetologia 2018; 61:2590-2597. https://doi.org/10.1007/s00125-018-4723-y.

8. Armstrong DG, Andrew JM, Boulton MD, Bus SA, Diabetic foot ulcers and their recurrence. New England Journal of Medicine 2017; 376:2367-2375. DOI: 10.1056/NEJMra1615439.

9. Monteiro-Soares M, Boyko EJ, Ribeiro J, Ribeiro I, Dinis-Ribeiro M, Predictive factors for diabetic foot ulceration: a systematic review 2012. Diabetes/Metabolism Research and Reviews 28:574-600. https://doi.org/10.1002/dmrr.2319.

10. Robbins JM, Strauss G, Aron D, Long J, Kuba J, Kaplan Y, Mortality rates and diabetic foot ulcers: Is it time to communicate mortality risk to patients with diabetic foot ulceration? J. Am Podiatr Med Assoc 2008; 98:489-493. https://doi.org/10.7547/0980489.

11. Rastogi A, Goyal G, Kesevan R, Bal A, Kumar H, Mangalanadanam PK, Jude EB, Armstrong DG, Bhansali $A$, Long term outcomes after incident diabetic foot ulcer: Multicenter large cohort prospective study (EDI-FOCUS investigators) epidemiology of diabetic foot complications study: Epidemiology of diabetic foot complications study. Diabetes Research and Clinical Practice 2020; 162:108113. https://doi.org/10.1016/j.diabres.2020.108113.

12. Stess RM, Jensen SR, Mirmiran R, The role of dynamic plantar pressures in diabetic foot ulcers. Diabetes Care 1997; 20:855-858. https://doi.org/10.2337/diacare.20.5.855.

13. Sawacha, Z, Guarneri, G, Cristoferi, G, Guiotto, A, Avogaro, A, Cobelli, C. Integrated kinematicskinetics-plantar pressure data analysis: a useful tool for characterizing diabetic foot biomechanics. Gait and Posture 2012; 36(1):20-26. https://doi.org/10.1016/j.gaitpost.2011.12.007. 
14. Halawa, MR, Eid YM, El-Hilaly RA, Abdelsalam MM, Relationship of planter pressure and glycaemic control in type 2 diabetic patients with and without neuropathy. Diabetes Metab Syndr 2018; 12:99-104. https://doi.org/10.1016/j.dsx.2017.09.010.

15. Pataky Z, Assal J-P, Conne P, Vuagnat H, Golay A, Plantar pressure distribution in type 2 diabetic patients without peripheral neuropathy and peripheral vascular disease. Diabetic Medicine 2005; 22:762-767. https://doi.org/10.1111/j.1464-5491.2005.01520.x.

16. Bacarin TA, Sacco ICN, Hennig EM, Plantar pressure distribution patterns during gait in neuropathy patients with a history of foot ulcers. Clinics 2009; 65(2):113-120. https://doi.org/10.1590/S1807-59322009000200008.

17. Fawzy, OA, Arafa A, El Wakeel, MA, Abdul Kareem, SH. Plantar pressure as a risk assessment tool for diabetic foot ulceration in Egyptian patients with diabetes. Clin Med Insights Endocrinol Diabetes 2014; 7:31-39. https://doi.org/10.4137/CMED.S17088.

18. Fernando ME, Crowther RG, Pappas E, Lazzarini PA, Cunningham M, Singh Sangla K, Buttner P, Golledge J, Plantar pressure in diabetic peripheral neuropathy patients with active foot ulceration, previous ulceration and no history of ulceration: A meta-analysis of observational studies. PLoS ONE 2014; 9(6):e99050. https://doi.org/10.1371/journal.pone.0099050.

19. Lee P-Y, Kong P-W, Pua Y-H, Reliability of peak foot pressure in patients with previous diabetic foot ulceration. Gait and Posture 2019; 70:6-11. https://doi.org/10.1016/j.gaitpost.2019.02.001.

20. Hellstrand Tang $U$, Foot deformities, function in the lower extremities, and plantar pressure in patients with diabetes at high risk to develop foot ulcers. Diabetic Foot and Ankle 2015; 6:27593. https://doi.org/10.3402/dfa.v6.27593.

21. Viswanathan V, Sivagami M, Seena R, Snehalatha C, Ramachandran A, Veves A, Increased forefoot to rearfoot plantar pressure ratio in South Indian patients with diabetic foot ulceration. Diabetic Medicine 2004; 21:395-396. https://doi.org/10.1111/j.1464-5491.2004.01135.x.

22. Searle Mosteo A, Spink MJ, Chuter VH, Validation of a weight bearing ankle equinus value in older adults with diabetes. Journal of Foot and Ankle Research 2018; 11:62. https://doi.org/10.1186/s13047-018-0306-x.

23. Jan Y-K, Lung C-W, Cuaderes E, Rong D, Boyce K, Effect of viscoelastic properties of plantar soft tissues on plantar pressures at the first metatarsal head in diabetics with peripheral neuropathy. Physiological Measurement 2013; 34:53-66. https://doi.org/10.1088/0967-3334/34/1/53.

24. Caselli A, Pham H, Giurini JM, Armstrong DG, Veves A, The forefoot-to-rearfoot plantar pressure ratio is increased in severe diabetic neuropathy and can predict foot ulceration. Diabetes Care 2002; 25:1066-1071. https://doi.org/10.2337/diacare.25.6.1066.

25. Wilson E, Understanding how the Achilles tendon affects plantar pressure. Podiatry Today 2009; 22:16-20.

26. Couppé C, Brüggebusch Svensson R, Kongsgaard M, Kovanen V, Grosset J, Snorgaard O et al., Human Achilles tendon glycation and function in diabetes. J Appl Physiol 2016; 120:130-137. https://doi.org/10.1152/japplphysiol.00547.2015. 
27. Bus SA, Foot structure and footwear prescription in diabetes mellitus. Diabetes/Metabolism Research and Reviews 2008; 24(Suppl 1):S90-S95. https://doi.org/10.1002/dmrr.840.

28. Branthwaite $H$, Chockalingam N, Greenhalgh A, The effect of shoe toe box shape and volume on forefoot interdigital and plantar pressures in healthy females $2013 ; 6: 28$. https://doi.org/10.1186/1757-1146-6-28.

29. Chatwin KE, Abbott CA, Boulton AJM, Bowling FL, Reeves ND, The role of foot pressure measurement in the prediction and prevention of diabetic foot ulceration - A Comprehensive review. Diabetes/Metabolism Research and Reviews 2019; e3258. https://doi.org/10.1002/dmrr.3258.

30. Moher D, Liberati A, Tetzlaff J, Altman DG, The PRISMA Group. Preferred Reporting Items for Systematic Reviews and Meta-Analyses: The PRISMA Statement. PLoS Med 2009; 6(7): e1000097. doi:10.1371/journal.pmed1000097.

31. Bus SA, Lavery LA, Monteiro-Soares M, Rasmussen A, Raspovic A, Sacco ISN, van Netten JJ, Guidelines on the prevention of foot ulcers in persons with diabetes (IWGDF 2019 update). Diabetes Metabolism/Research and Reviews 2020; 36(S1):e3269.

32. Ulbrecht JS, Cavanagh PR, Shoes and insoles for at-risk people with diabetes in Armstrong DG and Lavery LA, Clinical care of the diabetic foot (3rd edition). American Diabetes Association, pp.1617.

33. Ferber R, Webber T, Everett B, Groenland M, Validation of plantar pressure measurements for a novel in-shoe plantar sensory replacement unit 2013. Journal of Diabetes, Science and Technology 7(5):1167-1175. https://doi.org/10.1177/193229681300700535.

34. Bus SA, Waaijman R, Arts M, de Haart M, Busch-Westbroek T, van Baa J, Nollet F, Effect of custom-made footwear on foot ulcer recurrence in diabetes. Diabetes Care. 2013; 36:4109-4116. https://doi.org/10.2337/dc13-0996.

35. Preece SJ, Chapman JD, Braunstein B, Brüggemann G-P, Nester CJ, Optimisation of rocker sole footwear for prevention of first plantar ulcer: Comparison of group-optimised and individuallyselected footwear designs. Journal of Foot and Ankle Research 2017; 10:27. https://doi.org/10.1186/s13047-017-0208-3.

36. Lin T, Sheen H, Chung C, Yang S, Lin S, Luo H,, Chen C, Chan I, Shih H, Sheu WH, The effect of removing plugs and adding arch support to foam based insoles on plantar pressures in people with diabetic peripheral neuropathy. J. Foot and Ankle Research 2013; 6:29. https://doi.org/10.1186/1757-1146-6-29.

37. Abbott CA, Chatwin KE, Foden P, Hasan AN, Sange C, Rajbhandari SM et al., Innovative intelligent insole system reduces diabetic foot ulcer recurrence at plantar sites: A prospective, randomised, proof-of-concept study 2019. Lancet Digital Health 1(6):e308-e318. https://doi.org/10.1016/S25897500(19)30128-1.

38. De León Rodriguez D, Allet L, Golay A, Philippe J, Assal J-P, Hauert C-A, Pataky Z, Biofeedback can reduce foot pressure to a safe level and without causing new at-risk zones in patients with diabetes and peripheral neuropathy. Diabetes/Metabolism Research and Reviews 2013; 29:139-144. https://doi.org/10.1002/dmrr.2366. 
39. Waaijman R, Arts MLJ, Haspels R, Busch-Westbroek TE, Nollet F and Bus SA, Pressure-reduction and preservation in custom-made footwear of patients with diabetes and a history of plantar ulceration. Diabetic Medicine 2012; 29(12):1542-1549. https://doi.org/10.1111/j.14645491.2012.03700.x.

40. Waaijman R, Arts M, Haspels R, Busch T, Bus SA, Biomechanical optimization of newly prescribed therapeutic footwear in diabetic patients with a history of plantar ulceration. Conference of the International Society of Biomechanics (ISB) July 3-7, 2011 Brussels.

41. Owings TM, Apelqvist J, Stenström A, Becker M, Bus SA, Kalpen A, Ulbrect JS, Cavangh PR, Plantar pressures in diabetic patients with foot ulcers which have remained healed. Diabetic Medicine 2009; 26:1141-1146. https://doi.org/10.1111/j.1464-5491.2009.02835.x.

42. Arts MLJ, Waaijman R, de Haart M, Keukenkamp R, Nollet F, Bus SA, Offloading effect of therapeutic footwear in patients with diabetic neuropathy at high risk for plantar foot ulceration. Diabetic Medicine 2012; 29:1534-1541. https://doi.org/10.1111/j.1464-5491.2012.03770.x.

43. Arts MLJ, De Haart M, Waaijman R, Dahmen R, Berendsen H, Nollet F, Bus SA, Data-driven directions for effective footwear provision for the high-risk diabetic foot. Diabetic Medicine 2015; 32:790-797. https://doi.org/10.1111/dme.12741.

44. Bus SA, Haspels R, Busch-Westbroek TE, Evaluation and optimization of therapeutic footwear for neuropathic diabetic foot patients using in-shoe plantar pressure analysis 2011. Diabetes Care 34:1595-1600. https://doi.org/10.2337/dc10-2206.

45. Martinez-Santos A, Preece S, Nester CJ, Evaluation of orthotic insoles for people with diabetes who are at-risk of first ulceration. J. Foot and Ankle Research 2019; 12:35. https://doi.org/10.1186/s13047-019-0344-z.

46. Telfer S, Woodburn J, Collier A, Cavanagh PR, Virtually optimized insoles for offloading the diabetic foot: A randomized crossover study 2017. Journal of Biomechanics 60:157-161. https://doi.org/10.1016/j.jbiomech.2017.06.028.

47. Parker DJ, Nuttall GH, Vray N, Hugill T, Martinez-Santos A, Edwards RT, Nester C, A randomised controlled trial and cost-consequence analysis of traditional and digital foot orthoses supply chains in a National Health Service setting: Application to feet at risk of diabetic plantar ulceration. Journal of Foot and Ankle Research 2019; 12:2. https://doi.org/10.1186/s13047-018-0311-0.

48. Waaijman R, de Haart M, Arts MLJ, Wever D, Verlouw AJWE, Nollet F, Bus SA, Risk factors for plantar foot ulcer recurrence in neuropathic diabetic patients. Diabetes Care 2014; 37:1697-1705. https://doi.org/10.2337/dc13-2470.

49. Giacomozzi C, Uccioli L, Learning from experience: A simple effective protocol to test footwear prescriptions for the Diabetic foot by using the Pedar system. J. Biomedical Science and Engineering 2013; 6:45-57, 55. doi:10.4236/jbise.2013.65A008.

50. Chapman JD, Preece SJ, Nester CJ, Braunstein B, Höhne A, Brüggermann G-P, Evaluating the effect of apex position and rocker in curved rocker shoes. Journal of Foot and Ankle Research 2014; 7(Suppl 1):A66. 
51. Pataky Z, de Leon Rodriguez D, Allett L, Golay A, Assal M, Assal J-P, Hauert C-A, Biofeedback for foot offloading in diabetic patients with peripheral neuropathy. Diabetic Medicine 2010; 27:61-64. DOI: 10.1111/j.1464-5491.2009.02875.x.

52. Van GH, Haddad J, Bensimon Y, Dillard L, Jacobs D, Biofeedback strategy and reduction of foot pressure in diabetic patient with neuropathy: Benefit of connected insoles FEETME: a preliminary study. Ann Phys Rehabil Med. 2017; 60:e40.

53. Arts MLJ, Bus SA, Twelve steps per foot are recommended for valid and reliable in-shoe plantar pressure data in neuropathic diabetic patients wearing custom made footwear. Clinical Biomechanics 2011; 26:880-884. https://doi.org/10.1016/j.clinbiomech.2011.05.001.

54. Bus SA, Lavery LA, Monteiro-Soares M, Rasmussen A, Raspovic A, Sacco ISN, van Netten JJ, Guidelines on the prevention of foot ulcers in persons with diabetes (IWGDF 2019 update). Diabetes Metabolism/Research and Reviews 2020; 36(S1):e3269.

55. Branthwaite $H$, The effect of shoe toe box shape and volume on forefoot interdigital and plantar pressures in healthy females. Journal of Foot and Ankle Research 2013; 6:28. https://doi.org/10.1186/1757-1146-6-28.

56. Caselli A, The forefoot-to-rearfoot plantar pressure ratio is increased in severe diabetic neuropathy and can predict foot ulceration. Diabetes Care 2002; 25:1066 -1071.

57. Mondal A, Sen S, Chanda D, Kundu S, Chatterjee M, Mukherjee S, Evaluation of diabetic polyneuropathy in type 2 diabetes by nerve conduction study and association of severity of neuropathy with serum SFasL level. Indian J Endocrinol Metab 2012; 16(Suppl.2):S465-S467.

58. Rondinelli RD, Genovese E, Brigham CR, Association A M. Guides to the Evaluation of Permanent Impairment: American Medical Association; 2008.

59. Hardman RL, Overview of classification systems in peripheral artery disease. Semin Intervent Radiol. 2014; 31(4): 378-388.

60. Price C, Parker D, Nester C, Validity and repeatability of three in-shoe pressure measurement systems. Gait and Posture 2016; 46:69-74. https://doi.org/10.1016/j.gaitpost.2016.01.026.

61. Pataky Z, Faravel L, Da Silva J, Assal JP, A new ambulatory foot pressure device for patients with sensory impairment. A system for continuous measurement of plantar pressure and a feed-back alarm. Journal of biomechanics 2000; 33:1135-1137.

62. Jones P, Bibb R, Davies MJ, Khunti K, McCarthy M, Webb D and Zaccardi F, Prediction of Diabetic Foot Ulceration: The Value of Using Microclimate Arrays. J. Diabetes, Science and Technology 2020; 14(1):55-64. https://journals.sagepub.com/doi/full/10.1177/1932296819877194. 
SUPPLEMENAL TABLE $1 \quad$ Studies Comparing Plantar Pressure in People with Diabetes and Neuropathy (DN) with Healthy Controls (CG)

\begin{tabular}{|c|c|c|c|c|c|c|c|c|c|c|c|c|}
\hline \multirow[b]{2}{*}{ Paper } & \multirow[b]{2}{*}{ Year } & \multicolumn{5}{|c|}{ Participant Cohort } & \multirow[b]{2}{*}{ Region } & \multicolumn{5}{|c|}{ Pressure (Mean kPa for Region and Standard Deviation) } \\
\hline & & CG & D & DN & DNU & DNA & & CG & D & DN & DNU & DNA \\
\hline Bacarin [16] & 2008 & 20 & -- & 17 & 10 & -- & Mid-foot & $139.4 \pm 76.4$ & -- & $205.3 \pm 118.6$ & $290.7 \pm 151.5$ & -- \\
\hline Fawzy [17] & 2014 & -- & 37 & 33 & -- & 30 & Forefoot & -- & $292.8 \pm 83.9$ & $318.2 \pm 58.8$ & -- & $348.6 \pm 43.0$ \\
\hline Halawa [14] & 2018 & 30 & 30 & 20 & -- & -- & $\begin{array}{r}\text { Mid-foot (right) } \\
\text { Mid-foot (left) }\end{array}$ & $\begin{array}{l}82.1 \pm 30.8 \\
79.5 \pm 27.5\end{array}$ & $\begin{array}{l}104.9 \pm 40.9 \\
106.2 \pm 46.3\end{array}$ & $\begin{array}{l}138.2 \pm 76.3 \\
117.1 \pm 64.0\end{array}$ & -- & -- \\
\hline Sawacha[13] & 2011 & 12 & -- & 12 & -- & -- & Mid-foot & 312.3 & -- & 515.6 & -- & -- \\
\hline Pataky [15] & 2005 & 15 & -- & 15 & -- & - & $\begin{array}{r}\text { Hallux (right) } \\
\text { Hallux (left) } \\
\text { MTS5 (right) } \\
\text { MTS5 (left) }\end{array}$ & $\begin{array}{c}101 \pm 94 \\
104 \pm 43 \\
97 \pm 32 \\
91 \pm 42\end{array}$ & -- & $\begin{array}{l}205 \pm 94 \\
165 \pm 61 \\
160 \pm 68 \\
174 \pm 65\end{array}$ & $\begin{array}{ll}- \\
-\end{array}$ & -- \\
\hline Paper & Year & CG & D & DN & DNU & DNA & Region & \multicolumn{4}{|c|}{ Pressure (Mean $\mathbf{N} / \mathrm{cm}^{2}$ for Region and Standard Deviation) } & \\
\hline Stess [12] & 1997 & -- & 34 & 14 & 49 & $33^{*}$ & Forefoot & -- & $40.7 \pm 1.7$ & $40.5 \pm 2.8$ & $48.0 \pm 1.8$ & -- \\
\hline
\end{tabular}

$\underline{\text { Key }}$

Participant Cohort: $C G$ = Control group; $D=$ Diabetes without neuropathy or history of ulceration; $D N=$ Diabetes and neuropathy; DNU = Diabetes, neuropathy and a history of ulceration; DNA = Diabetes, neuropathy and an active ulcer where in Bacarin, DNA=33 is a subset of DNU=49.

Region: Only the mid-foot peak plantar pressure was found to be statistically significant - see Bacarin's table 2 and Sawacha's table 3

Pressure: $\mathrm{kPa}=$ Kilopascals. $\mathrm{N} / \mathrm{cm}^{2}=$ Newtons for a defined area in squared centimetres 
SUPPLEMENTAL TABLE 2 Previous ulcer locations (where known) within in-shoe pressure studies

\begin{tabular}{|c|c|c|c|c|c|c|c|c|c|c|c|c|}
\hline Paper & Year & Total & Hallux & $\begin{array}{l}\text { Toes } \\
2 / 3\end{array}$ & $\begin{array}{l}\text { Toes } \\
4 / 5\end{array}$ & $\begin{array}{l}\text { MTH } \\
1 \\
\end{array}$ & $\begin{array}{l}\text { MTH } \\
2 / 3\end{array}$ & $\begin{array}{l}\text { MTH } \\
4 / 5 \\
\end{array}$ & $\begin{array}{l}\text { Medial } \\
\text { Midfoot }\end{array}$ & $\begin{array}{l}\text { Lateral } \\
\text { Midfoot }\end{array}$ & $\begin{array}{l}\text { MTH } \\
\text { Base }\end{array}$ & Heel \\
\hline Arts & 2012 & 147 & $\begin{array}{l}(34) \\
23.1 \% \\
\end{array}$ & $\begin{array}{l}(23) \\
15.6 \%\end{array}$ & $\begin{array}{l}(2) \\
1.4 \%\end{array}$ & $\begin{array}{l}(39) \\
26.5 \%\end{array}$ & $\begin{array}{l}(20) \\
13.6 \%\end{array}$ & $\begin{array}{l}(17) \\
11.6 \%\end{array}$ & $\begin{array}{l}(5) \\
3.4 \%\end{array}$ & $\begin{array}{l}(2) \\
1.4 \%\end{array}$ & $\begin{array}{l}(4) \\
2.7 \% \\
\end{array}$ & $\begin{array}{l}1) \\
0.7 \% \\
\end{array}$ \\
\hline Arts & 2015 & 139 & $38.1 \%$ & $2.9 \%$ & -- & $28.1 \%$ & $19.4 \%$ & $10.8 \%$ & $0.7 \%$ & -- & -- & -- \\
\hline Bus & 2011 & 18 & $\begin{array}{l}(3) \\
16.7 \%\end{array}$ & \multicolumn{2}{|l|}{$\begin{array}{l}1) \\
5.5 \%\end{array}$} & \multicolumn{3}{|l|}{$\begin{array}{l}(11) \\
61.1 \%\end{array}$} & \multicolumn{2}{|l|}{$\begin{array}{l}(3) \\
16.7 \%\end{array}$} & -- & -- \\
\hline Owings & 2009 & -- & $x$ & -- & -- & $x$ & -- & -- & -- & -- & & \\
\hline Waaijman & 2012 & 85 & \begin{tabular}{|l|}
$(15)$ \\
$17.6 \%$ \\
\end{tabular} & $\begin{array}{l}(17) \\
20.0 \%\end{array}$ & $\begin{array}{l}2) \\
2.4 \% \\
\end{array}$ & $\begin{array}{l}(23) \\
27.1 \%\end{array}$ & $\begin{array}{l}15) \\
17.6 \% \\
\end{array}$ & $\begin{array}{l}11) \\
12.9 \%\end{array}$ & $\begin{array}{l}1) \\
1.2 \% \\
\end{array}$ & $\begin{array}{l}1) \\
1.2 \% \\
\end{array}$ & -- & -- \\
\hline Waaijman* & 2014 & 147 & $\begin{array}{l}(34) \\
23.1 \%\end{array}$ & $\begin{array}{l}(23) \\
15.6 \%\end{array}$ & $\begin{array}{l}(2) \\
1.4 \%\end{array}$ & $\begin{array}{l}(39) \\
26.5 \%\end{array}$ & $\begin{array}{l}(20) \\
13.6 \%\end{array}$ & $\begin{array}{l}(17) \\
11.6 \%\end{array}$ & $\begin{array}{l}\text { (5) } \\
3.4 \%\end{array}$ & $\begin{array}{l}2) \\
1.4 \%\end{array}$ & $\begin{array}{l}(4) \\
2.7 \%\end{array}$ & $\begin{array}{l}11) \\
0.7 \%\end{array}$ \\
\hline
\end{tabular}

Numbers of ulcers at each location shown in brackets; and percentages of ulcers at each location. Where the number or percentage of ulcers at each location is not known, a marker is provided to indicate the locations where ulcers are known to have occurred.

Where these figures are split into sub-totals, IF = Improved Footwear, UC = Usual Care.

*171 people with healed ulcers but only 147 ulcer locations given amputations.

Abbott missing given split into both intervention group/control group and left/right feet. 
SUPPLEMENTAL TABLE 3

\begin{tabular}{|l|l|l|l|l|l|l|}
\hline & & \multicolumn{2}{|l|}{ Standardisation } & & \multicolumn{2}{l|}{ Pressure Measurement } \\
\hline Paper & Year & Socks & Insoles & Sensor & Sampling Rate & Intervention \\
\hline Abbott & 2019 & $x$ & $x$ & SurroSense & $8 \mathrm{~Hz}$ & Biofeedback (Real-time) \\
\hline Arts & 2012 & $\checkmark$ & $\checkmark$ & Pedar-X & $50 \mathrm{~Hz}$ & Footwear evaluation \\
\hline Arts & 2015 & $\checkmark$ & $\checkmark$ & Pedar-X & $50 \mathrm{~Hz}$ & Footwear evaluation \\
\hline Bus & 2011 & $x$ & $\checkmark$ & Pedar-X & $50 \mathrm{~Hz}$ & Footwear evaluation \\
\hline Bus & 2013 & $x$ & $\checkmark$ & Pedar-X & $50 \mathrm{~Hz}$ & Outcomes from Threshold \\
\hline Chapman & 2014 & -- & -- & -- & -- & Footwear evaluation \\
\hline De León Rodriguez & 2013 & $x$ & $x$ & Pedar-X & $50 \mathrm{~Hz}$ & Biofeedback \\
\hline Giacomozzi & 2013 & $x$ & $\checkmark$ & Pedar-X & $50 \mathrm{~Hz}$ & Footwear evaluation \\
\hline Lin & 2013 & $\checkmark$ & $Q$ & Pedar-X & $50 \mathrm{~Hz}$ & Insole evaluation / arch support \\
\hline Martinez-Santos & 2019 & $x$ & $Q$ & Pedar-X & $50 \mathrm{~Hz}$ & Insole evaluation \\
\hline Owings & 2009 & $x$ & $x$ & Pedar & -- & Pressures in Remission \\
\hline Parker & 2019 & $x$ & $\checkmark$ & Pedar & -- & Orthoses evaluation \\
\hline Pataky & 2010 & -- & -- & Pedar & $100 \mathrm{~Hz}$ & Biofeedback \\
\hline Preece & 2017 & $x$ & $x$ & Pedar-X & $50 \mathrm{~Hz}$ & Footwear evaluation \\
\hline Searle & 2018 & $\checkmark$ & -- & Pedar-X & $100 \mathrm{~Hz}$ & Equinus \& pressure analysis \\
\hline Van & 2017 & -- & -- & Feetme & -- & Biofeedback \\
\hline Waaijman & 2011 & $x$ & $\checkmark$ & Pedar-X & -- & Footwear evaluation \\
\hline Waaijman & 2012 & $\checkmark$ & $\checkmark$ & Pedar-X & $50 \mathrm{~Hz}$ & Footwear evaluation \\
\hline Waaijman & 2014 & $x$ & $\checkmark$ & Pedar-X & $50 \mathrm{~Hz}$ & Risk factor evaluation \\
\hline
\end{tabular}

Key

$Q=$ The subject of evaluation e.g. footwear, insole etc. $x=$ Not standardised. $\checkmark=$ Standardised $--=$ unspecified. 
SUPPLEMENTAL TABLE 4

\begin{tabular}{|c|c|c|c|c|c|c|c|c|c|c|c|c|c|c|c|c|c|c|c|c|c|}
\hline Paper and year & $\begin{array}{l}\text { Test } \\
\text { Method } 1\end{array}$ & $\begin{array}{l}\text { No. } \\
\text { Sites }\end{array}$ & 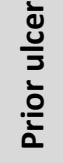 & $\underset{\frac{x}{\sqrt{T}}}{\stackrel{x}{I}}$ & $\stackrel{r}{\underline{r}}$ & 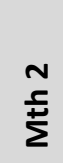 & $\begin{array}{l}m \\
\stackrel{m}{c} \\
\dot{\sum}\end{array}$ & $\begin{array}{l}\dot{2} \\
\stackrel{5}{\Sigma} \\
\Sigma\end{array}$ & $\sum_{\substack{n \\
\sum}}^{n}$ & $\frac{\overline{\bar{g}}}{\overline{\bar{N}}}$ & $\begin{array}{l}+ \\
\stackrel{0}{0} \\
\frac{0}{0} \\
\dot{z}\end{array}$ & $\begin{array}{l}\bar{\Phi} \\
\stackrel{\Phi}{I}\end{array}$ & 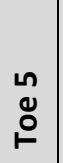 & $\frac{\frac{2}{2}}{\frac{0}{0}}$ & Cond & $\begin{array}{l}\text { Test } \\
\text { Method } 2\end{array}$ & $\begin{array}{l}\text { No. } \\
\text { Sites }\end{array}$ & $\stackrel{\frac{x}{3}}{\frac{\underline{\pi}}{\underline{T}}}$ & $\begin{array}{l}\frac{\pi}{\bar{N}} \\
\stackrel{0}{\pi} \\
0\end{array}$ & $\frac{\frac{0}{x}}{\frac{x}{c}}$ & $\frac{\grave{d}}{\stackrel{ \pm}{ \pm}}$ \\
\hline Abbott 2019 [37] & $\mathrm{M} / 10 \mathrm{~g}$ & 1 of 3 & & & $x$ & & $x$ & & $x$ & & & & & & & & & & & & \\
\hline Arts 2012 [42] & $\mathrm{M} / 10 \mathrm{~g}$ & 1 of 3 & & $x$ & $\mathrm{x}$ & & & & $x$ & & & & & & OR & $\mathrm{V} / \geq 25 \mathrm{~V}$ & 1 of 1 & $\mathrm{x}$ & & & \\
\hline Arts 2015 [43] & $\mathrm{M} / 10 \mathrm{~g}$ & 1 of 3 & & $x$ & $x$ & & & & $x$ & & & & & & OR & $\mathrm{V} / \geq 25 \mathrm{~V}$ & 1 of 1 & $\mathrm{x}$ & & & \\
\hline Bus 2011 [44] & $\mathrm{M} / 10 \mathrm{~g}$ & 1 of 6 & & $x$ & $x$ & & $x$ & & $x$ & & $\mathrm{x}$ & $\mathrm{x}$ & & & & & & & & & \\
\hline Bus 2013 [34] & $\mathrm{M} / 10 \mathrm{~g}$ & 1 of 3 & & $x$ & $x$ & & & & $x$ & & & & & & OR & $\mathrm{V} / \geq 25 \mathrm{~V}$ & 1 of 1 & $x$ & & & \\
\hline Chapman, 2014 [50] & -- & -- & -- & -- & -- & -- & -- & -- & -- & -- & -- & -- & -- & -- & -- & -- & -- & -- & -- & -- & -- \\
\hline $\begin{array}{l}\text { De León Rodriguez } \\
2013 \text { [38] }\end{array}$ & TF / $128 \mathrm{~Hz}$ & 4 of 8 & & $x$ & & & & & & $x$ & & & & & AND & $\mathrm{P} /$ absence $^{1}$ & & & $x$ & $x$ & \\
\hline Ferber, 2013 [33] & & & \multicolumn{12}{|c|}{ Healthy participants. People with neuropathy excluded } & & & & & & & \\
\hline $\begin{array}{l}\text { Giacomozzi² } 2013 \\
\text { [49] }\end{array}$ & NDS $6 / 10$ & $\mathrm{~N} / \mathrm{A}$ & \multicolumn{12}{|c|}{ Neuropathy disability score $>6 / 10$} & AND & $\begin{aligned} V / & \geq 25 \mathrm{~V}+ \\
& >20 \mathrm{~V}\end{aligned}$ & 2 of 2 & $\mathrm{x}$ & & & \\
\hline Lin 2013 [36] & $\mathrm{M} / 10 \mathrm{~g}$ & 1 of 6 & & $\mathrm{x}$ & $\mathrm{x}$ & $\mathrm{x}$ & $\mathrm{x}$ & $\mathrm{x}$ & $\mathrm{x}$ & & & & & & AND & TF / $128 \mathrm{~Hz}$ & 2 of 6 & \multirow{2}{*}{\multicolumn{4}{|c|}{$\begin{array}{l}\text { Unclear }^{3} \\
\text { Hallux,MTH1+5, } \\
\text { malleoli }\end{array}$}} \\
\hline $\begin{array}{l}\text { Martinez-Santos } \\
2019 \text { [45] }\end{array}$ & $\mathrm{M} / 10 \mathrm{~g}$ & 1 of 10 & \multicolumn{12}{|c|}{$\begin{array}{l}\text { Hallux, toes } 3+5, \text { MTH } 1+3+5 \text {, medial \& lateral arches, heel } \\
\text { and dorsum between toes } 1 \& 2\end{array}$} & OR & TF / $128 \mathrm{~Hz}$ & 1 of 4 & & & & \\
\hline Owings 2009 [41] & $\mathrm{M} / 10 \mathrm{~g}$ & 1 of 1 & $\mathrm{x}$ & & & & & & & & & & & & & & & & & & \\
\hline Parker 2019 [47] & $\mathrm{M} / 10 \mathrm{~g}$ & 3 of 10 & \multicolumn{12}{|c|}{ Sites at foot and ankle unspecified } & & & & & & & \\
\hline Pataky, $2010[51]$ & TF / $128 \mathrm{~Hz}$ & $\leq 4$ of 8 & & $\mathrm{x}$ & & & & & & $\mathrm{x}$ & & & & & AND & $\begin{array}{c}\text { Absence of } \\
\text { reflexes }\end{array}$ & -- & & $x$ & $x$ & \\
\hline Preece, 2017 [35] & $\mathrm{M} / 10 \mathrm{~g}$ & -- & & $\mathrm{x}$ & $\mathrm{x}$ & & & & $x$ & & & & $\mathrm{x}$ & $\mathrm{x}$ & & & & & & & \\
\hline Searle, 2018 [22] & $\mathrm{M} / 10 \mathrm{~g}$ & 1 of 4 & & $x$ & $x$ & & $x$ & & $\mathrm{x}$ & & & & & & OR & $\mathrm{V} />25 \mathrm{~V}$ & 1 of 1 & $\mathrm{x}$ & & & \\
\hline Telfer 2017 [46] & $\mathrm{M} / 10 \mathrm{~g}$ & $\geq 1$ & \multicolumn{12}{|c|}{ Sites / No. of sites unspecified } & & & & & & & \\
\hline Van, 2017 [52] & -- & -- & -- & -- & -- & -- & -- & -- & -- & -- & -- & -- & -- & -- & -- & -- & -- & -- & -- & -- & -- \\
\hline Waaijman, 2011 [40] & -- & -- & -- & -- & -- & -- & -- & -- & -- & -- & -- & -- & -- & -- & -- & -- & -- & -- & -- & -- & -- \\
\hline Waaijman 2012 [39] & $\mathrm{M} / 10 \mathrm{~g}$ & 1 of 3 & & $x$ & $x$ & & & & $x$ & & & & & & OR & $\mathrm{V} />25 \mathrm{~V}$ & 1 of 1 & $x$ & & & \\
\hline Waaijman 2014 [48] & $\mathrm{M} / 10 \mathrm{~g}$ & 1 of 1 & & $x$ & & & & & & & & & & & OR & $\begin{array}{l}\mathrm{V} / \text { Mean } \\
>25 \mathrm{~V}\end{array}$ & 3 & $x$ & & & $x$ \\
\hline
\end{tabular}

Key

Method: M (Monofilament), V (Vibration perception threshold), TF (Tuning fork), P (patella and ankle reflexes); NDS (Neuropathy disability score), -- method used is 
unspecified.

${ }^{1}$ Neuropathy is determined from patellar and ankle reflexes and a tuning fork used at the hallux and malleoli of both feet.

${ }^{2}$ Vibration perception threshold measured at the hallux of both feet, with two thresholds: $25 \mathrm{~V}$ for the first foot and $20 \mathrm{~V}$ for the second.

${ }^{3}$ Lin: Possibly the same six sites as with the monofilament although methodology cited involves dorsum of the first toe and dorsal aspect of the distal phalanx tested twice on each toe.

${ }^{4}$ Various $=$ Toes 1,3,5; Metatarsal heads 1,3,5, medial and lateral arches, heel and dorsum between toes 1 and 2.

\section{SUPPLEMENTAL TABLE $5 \quad$ PVD and re-ulceration within in-shoe pressure studies}

\begin{tabular}{|l|l|l|}
\hline Paper & Year & PVD related data \\
\hline Abbott & 2019 & $\begin{array}{l}\text { This study excluded severe vascular disease. No ABI. } \\
\text { Peripheral arterial status was assessed by palpating the dorsalis } \\
\text { pedis and posterior tibial pulses on both feet: } \\
\text { Palpable dorsalis pedis pulse } \\
\text { Left 89\% control group; 87\% intervention group } \\
\text { Right 83\% control group; 79\% intervention group }\end{array}$ \\
$\begin{array}{l}\text { Palpable dorsalis posterior tibial pulse } \\
\text { Left 83\% control group; 70\% intervention group } \\
\text { Right 83\% control group; 69\% intervention group }\end{array}$ \\
\hline Bus & $\begin{array}{l}\text { No further analysis on impact of presence of PVD on re-ulceration. } \\
\text { Peripheral arterial status was assessed according to the PEDIS } \\
\text { (perfusion, extent/size, depth/tissue loss, infection, sensation) } \\
\text { classification. Peripheral arterial disease was confirmed present } \\
\text { when pedal pulses were nonpalpable and the ankle-brachial index } \\
\text { was 0.9 in the foot with the most recent episode of ulceration } \\
\text { according to the PEDIS classification. In five patients, peripheral } \\
\text { arterial disease could not be assessed, and in six, data were missing } \\
\text { (n=160/171). }\end{array}$ \\
\hline \begin{tabular}{l} 
Peripheral artery disease: \\
\hline
\end{tabular}
\end{tabular}




\begin{tabular}{|l|l|l|}
\hline & & $\begin{array}{l}\text { Improved footwear 28.8\% } \\
\text { Usual care 37.5\% } \\
\text { No further analysis on impact of presence of PVD on re-ulceration. }\end{array}$ \\
\hline Owings & 2009 & $\begin{array}{l}\text { Exclusion criteria included major vascular disease. } \\
\text { "Only patients aged 30-80 years with ulcers that were of } \\
\text { predominantly neuropathic aetiology and who had remained } \\
\text { healed for at least 90 days were included." }\end{array}$ \\
\hline Waaijman & $\begin{array}{l}\text { Grade II peripheral arterial disease (\%): } \\
\text { Presence of peripheral arterial disease (grade I = no; grade II = yes) } \\
\text { was assessed using the Perfusion Extent Depth Infection Sensation } \\
\text { (PEDIS) classification. } \\
\text { All patients: 35.2 } \\
\text { All ulcer recurrences (model 1): } \\
\text { Patients with no ulcer 38.5 } \\
\text { Patients with ulcer 30.4 } \\
\text { Ulcer recurrences from unrecognized repetitive trauma (model 2): } \\
\text { Patients with no ulcer 36.5 } \\
\text { Patients with ulcer 30.8 } \\
\text { Univariate model (All ulcer recurrences model 1): } \\
\text { Peripheral arterial disease, grade II (vs. grade I) } \\
\text { OR: 0.78 (0.53-1.15) (95\% CI) P: 0.206 } \\
\text { Univariate model (Ulcer recurrences from unrecognized repetitive } \\
\text { trauma (model 2)) } \\
\text { OR: 0.79 (0.37-1.68) (95\% CI) P: 0.540 } \\
\text { "The inclusion of a selected group of only high-risk patients may also }\end{array}$ \\
& & \\
& 2014 \\
\hline
\end{tabular}




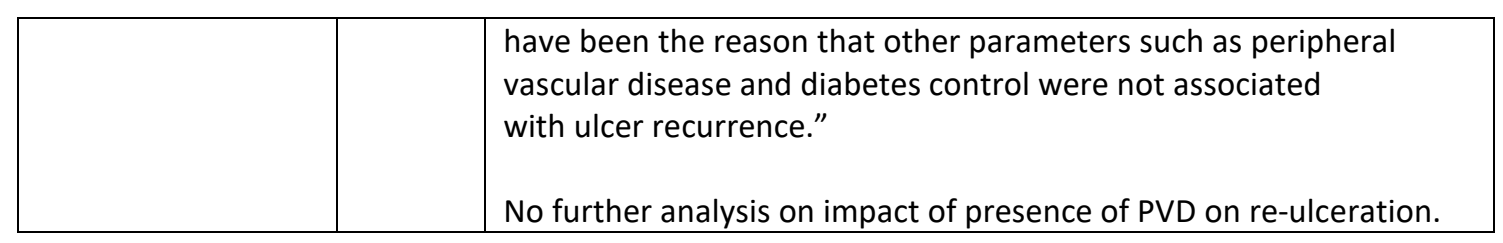

\section{SUPPLEMENTAL TABLE 6 Severity of neuropathy and re-ulceration within in-shoe pressure studies}

\begin{tabular}{|c|c|c|}
\hline Paper & Year & Severity of neuropathy related data \\
\hline Abbott & 2019 & $\begin{array}{l}\text { Severe (Neuropathy Disability Score: NDS } 9 \text { or 10): } \\
\text { Control group } 46 \% \\
\text { Intervention group } 44 \% \\
10 \text { g Semmes-Weinstein monofilament, abnormal test: } \\
\text { Left foot: control group } 76 \% \text {; intervention group } 91 \% \\
\text { Right foot: control group 68\%; intervention group } 97 \% \\
\text { Neuropad, abnormal test: } \\
\text { Control group: } 96 \% \\
\text { Intervention group: } 92 \% \\
\text { No further analysis on impact of presence of severe neuropathy on } \\
\text { re-ulceration. }\end{array}$ \\
\hline Bus & 2013 & $\begin{array}{l}\text { Inclusion criteria included participants with loss of protective foot } \\
\text { sensation as a result of peripheral neuropathy. } \\
\text { Loss of protective sensation based on vibration perception threshold } \\
>25 \text { volts } \\
\text { Improved footwear: } 85.2 \% \text {; Usual care: } 85.9 \% \\
\text { Vibration perception threshold (volts): } \\
\text { Improved footwear: } 50.0 \text { mean (11.1 SD); Usual care: } 50.0 \text { mean }(9.0 \\
\text { SD) }\end{array}$ \\
\hline Owings & 2009 & No apparent discussion of severity of neuropathy within cohort. \\
\hline
\end{tabular}




\begin{tabular}{|l|l|l|}
\hline Waaijman & 2014 & $\begin{array}{l}\text { Univariate model (All ulcer recurrences model 1): } \\
\text { Vibration perception threshold (volt) } \\
\text { OR: } 0.98(0.95-1.01 \mathrm{CI}) \mathrm{P}: 0.267\end{array}$ \\
& $\begin{array}{l}\text { Ulcer recurrences from unrecognized repetitive trauma (model 2): } \\
\text { Vibration perception threshold (volt) } \\
\text { OR: } 0.99 \text { (0.96-1.03 CI) P: } 0.662\end{array}$ \\
& "All patients had neuropathy and as a result already a high vibration \\
& perception threshold, which limits its potential to discriminate in \\
& logistic regression analysis".
\end{tabular}


Supplemental Figure 1

Examples of algorithms utilising the $200 \mathrm{kPa}$ threshold in offloading

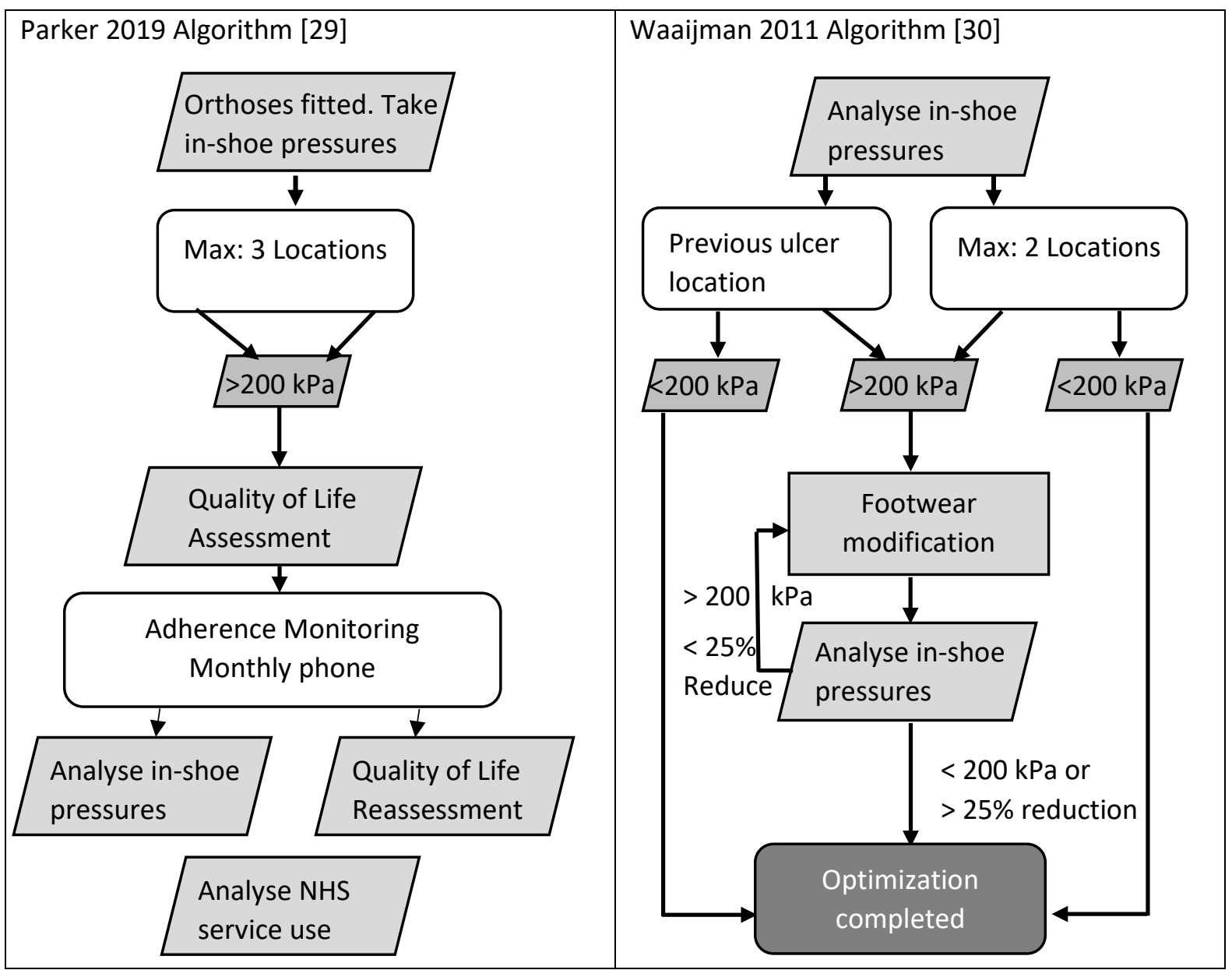

\section{Appendix A Search strings}

"pressure" AND "foot" AND "in-shoe" AND "diabetes" AND "threshold"

"plantar" AND "pressure" AND "foot" AND "in-shoe" AND "diabetes" AND "threshold" 